\title{
SPIN-CHARGE SEPARATION, CONFORMAL COVARIANCE AND THE SU(2) YANG-MILLS THEORY
}

\author{
Ludvig Faddeev* \\ S. Petersburg Branch of Steklov Mathematical Institute, \\ Russian Academy of Sciences, Fontanka 2\%, St. Petersburg, Russia \\ Antti J. Niemit \\ Department of Theoretical Physics, Uppsala University, \\ P.O. Box 803, S-75108, Uppsala, Sweden \\ Laboratoire de Mathematiques et Physique Theorique CNRS UMR 6083, \\ Université de Tours, Parc de Grandmont, F37200, Tours, France and \\ Chern Institute of Mathematics, Tianjin 3000\%1, P.R. China
}

(Dated: March 15, 2018) 


\begin{abstract}
In the low energy domain of four-dimensional $\mathrm{SU}(2)$ Yang-Mills theory the spin and the charge of the gauge field can become separated from each other. The ensuing field variables describe the interacting dynamics between a version of the $O(3)$ nonlinear $\sigma$-model and a nonlinear Grassmannian $\sigma$-model, both of which may support closed knotted strings as stable solitons. Lorentz transformations act projectively in the $O(3)$ model which breaks global internal rotation symmetry and removes massless Goldstone bosons from the particle spectrum. The entire Yang-Mills Lagrangian can be recast into a generally covariant form with a conformally flat metric tensor. The result contains the Einstein-Hilbert Lagrangian together with a nonvanishing cosmological constant, and insinuates the presence of a novel dimensionfull parameter in the Yang-Mills theory.
\end{abstract}

*Electronic address: Faddeev@pdmi.ras.ru

${ }^{\dagger}$ Electronic address: Antti.Niemi@teorfys.uu.se URL: http://www.teorfys.uu.se/people/antti 


\section{INTRODUCTION}

Apparently the necessity of a mass gap in a pure Yang-Mills theory and the nature of its particle spectrum were originally posed as problems by Wolfgang Pauli during a 1954 Princeton seminar by C.N. Yang [1]. However, despite over 50 years of effords the physical particle content of a pure, interacting four dimensional Yang-Mills theory remains a mystery. In particular, the explanation of color confinement is a theoretical challenge [2]. Dimensional transmutation with its intimate relationship to the high energy asymptotic freedom does open a door for a dimensionfull parameter to enter. But since the high energy limit of the Yang-Mills theory describes asymptotically free, massless gauge particles the precise relationship between this dimensionfull parameter and the mass gap which secures confinement remains unclear.

During the last ten years [3]-[5] we have investigated the possibility that the low energy spectrum of pure Yang-Mills theory could comprise of closed and knotted strings as stable solitons. This proposal is very natural from the point of view of QCD phenomenology. If quarks are indeed confined by stringlike collective excitations of the gauge field, in the absence of quarks these strings should close on themselves into stable and in general knotted and linked configurations. In [5] we proposed how the properties of such closed stringlike solitons could be related to the spectrum of the Yang-Mills theory. In particular, we suggested that in the case of a $S U(2)$ gauge theory the effective low energy Lagrangian should relate to the following version of the $O(3)$ nonlinear $\sigma$-model, originally proposed by one of us [6]

$$
\mathcal{L}_{\text {eff }}=\frac{\mathrm{m}^{2}}{2}\left(\partial_{a} \boldsymbol{s}\right)^{2}+\frac{1}{4}\left(\boldsymbol{s} \cdot \partial_{a} \boldsymbol{s} \times \partial_{b} \boldsymbol{s}\right)^{2}+V(\boldsymbol{s})
$$

Here $s$ is a three-component unit vector, and $\mathrm{m}$ is a parameter with the dimensions of mass. The last term is a potential term. It breaks the global $O(3)$ symmetry which is present in the first two terms, removing the two massless Goldstone bosons from the spectrum. Both numerical simulations [7] and formal mathematical arguments [8] have confirmed our proposal [9] that (11) does indeed support closed knotted strings as stable 
solitons.

Several approaches have been suggested, how to derive (11) directly from the YangMills theory. These derivations are commonly based on the following, incomplete Liealgebra expansion of the $S U(2)$ gauge field [3]

$$
\boldsymbol{A}_{a}=C_{a} \boldsymbol{s}+\partial_{a} \boldsymbol{s} \times \boldsymbol{s}+\rho \partial_{a} \boldsymbol{s}+\sigma \partial_{a} \boldsymbol{s} \times \boldsymbol{s}
$$

where $\rho$ and $\sigma$ are real scalars. The first two terms in (2) were originally introduced by Duan and Ge [10], and subsequently by Cho [11], to describe the properties of Wu-Yang monopoles. Indeed, this is a very natural decomposition of the gauge field in terms of the $S U(2)$ Lie algebra. When (2) is substituted to the Yang-Mills Lagrangian, one finds that the structure (10) emerges after one-loop radiative corrections are taken into account [12].

The apparent lack of off-shell completeness in (2) prompted us in [4] to propose an alternative decomposition of the gauge field. This new decomposition is off-shell complete, and (2) assumes a role in ensuring its manifest gauge covariance. Our new decomposition leads to many unexpected consequences, some of which we shall reveal in the present article. In particular, it admits a very intriguing physical interpretation: The decomposition [4] is intimately related to the slave-boson decomposition [13] that has been introduced independently in the context of strongly correlated electron systems as an alternative to Cooper pairing and Higgs effect [14]. As a consequence our new decomposition suggests that the separation between the spin and the charge could be a general phenomenon that can be exhibited by a large variety of quantum fields [15], [16].

A common feature of these spin-charge decompositions is that they all seem to involve a real-valued scalar field. A nonvanishing ground state expectation value for this scalar field describes a condensate. This corresponds to the density of the material environment, and the presence of a nontrivial condensate is a necessary condition for the spin-charge decomposition to occur. The condensate can also yield an alternative to the conventional Higgs effect [14]. 
Here we find that in the case of $S U(2)$ Yang-Mills theory the real-valued scalar field admits another interpretation. It is the conformal scale of a metric tensor that describes a conformally flat space-time. In terms of the spin-charge separated variables, the YangMills Lagrangian then contains both the Einstein-Hilbert Lagrangian and a cosmological constant. Since conformal flatness is equivalent to the vanishing of the traceless Weyl (conformal) tensor $W_{\mu \nu \rho \sigma}$, the gravitational contribution to the Yang-Mills Lagrangian can be interpreted as the $\gamma \rightarrow \infty$ limit of the higher derivative gravitational Lagrangian

$$
\mathcal{L}_{E H}=\frac{1}{\kappa} \sqrt{g} R-\Lambda \cdot \sqrt{g}+\gamma \cdot W_{\mu \nu \rho \sigma}^{2}
$$

It has been shown 17] that the Lagrangian (3) is part of a renormalizable higher derivative quantum theory of gravity. In particular, the one-loop $\beta$-function for $\gamma$ does indeed send this coupling to infinity in the short distance limit. This enforces asymptotically the condition

$$
W_{\mu \nu \rho \sigma} \sim 0
$$

Hence at short distances the space-time becomes asymptotically (locally) conformally flat. We also note that the presence of the higher derivative Weyl tensor contribution in (13) gives rise to a linearly increasing component in the large distance interactions. Furthermore, our reformulation of the Yang-Mills theory in a manner that contains the Einstein-Hilbert and cosmological constant terms has the additional peculiar consequence that a novel dimensionfull parameter enters the Yang-Mills Lagrangian.

We start the present article by defining our notations. We then proceed to describe the new decomposition [4] of the four dimensional $S U(2)$ gauge field. This decomposition singles out the Cartan direction of the $S U(2)$ Lie algebra. This leaves us with a geometric structure that involves the Grassmannian manifold $G(4,2)$ of two dimensional planes that are embedded in a four dimensional space. The Grassmannian geometry guides our subsequent decomposition of the gauge field into its spin and charge constituents. In particular, it leads us to a $O(3)$ symmetric order parameter, a three component internal space unit vector field $\boldsymbol{n}$ akin the vector field $s$ in (11). 
The Grassmannian structure also introduces an internal, compact $U(1)$ gauge symmetry. In the context of lattice gauge theories [18] a compact $U(1)$ gauge theory is known to display a first-order phase transition between a strong coupling phase and a weak coupling phase. The strong coupling phase exhibits confinement, which is absent in the weak coupling phase.

It is quite natural to expect that in an abelian theory the gauge coupling decreases when the distance increases. Thus the presence of the internal compact $U(1)$ gauge structure may explain why in short distance Yang-Mills theory the spin and the charge can become strongly confined into asymptotically free and pointlike gauge particles, and why the decomposition into independent spin and charge carriers can only occur in the weakly coupled long-distance domain of the compact $U(1)$ theory.

The internal $U(1)$ gauge structure leads to a projective realization of the Lorentz transformations in the $O(3)$ unit vector $\boldsymbol{n}$. The ensuing one-cocycle breaks the global $O(3)$ symmetry which is displayed by the first two terms in the corresponding Lagrangian (11), even in the absence of an explicit symmetry breaking term such as the third term in (11): Since the ground state of the theory can not violate Lorentz invariance, the ground state direction of the vector $\boldsymbol{n}$ in the internal space becomes uniquely fixed. As a consequence the requirement that the ground state is Lorentz invariant, removes the two massless Goldstone bosons that are otherwise associated with the breaking of the global $O(3)$ symmetry in the dynamics of $\boldsymbol{n}$.

We then proceed to inspect the detailed structure of the Yang-Mills Lagrangian in terms of the separate spin and charge variables. In particular, we explain how the Lagrangian (11) for the internal vector $\boldsymbol{n}$ is embedded in the tree-level Yang-Mills Lagrangian, even before any radiative corrections are taken into account. Since (1) supports closed knotted strings as stable solitons 9], 7], 8], this endorses our proposal that knotted and closed stringlike solitons are indeed the natural candidates for describing the interacting spectrum of the pure $S U(2)$ Yang-Mills theory [5].

It turns out that the functional form (11) relates both to the spin and the charge degree 
of freedom. This suggests the presence of some kind of dual structure between the spin and charge variables in the Yang-Mills theory. Moreover, since the spin conventionally relates to magnetism while the charge relates to electricity, this could be the sought-after electric-magnetic duality of the Yang-Mills theory.

We then argue that our decomposition is independent of the way how we choose the Cartan direction in the $S U(2)$ Lie algebra. The gauge covariance becomes manifest when we introduce the structure of (2) in our decomposed gauge field.

We show that in terms of the spin and the charge variables the entire Yang-Mills Lagrangian admits a manifestly generally covariant form with a conformally flat metric tensor. Thus the $S U(2)$ Yang-Mills theory describes the interactions between (1D) and the $G(4,2)$ Grassmannian nonlinear $\sigma$-model in a conformally flat space-time. Both the Einstein-Hilbert Lagrangian and a cosmological constant term are present. This also introduces a dimensionfull parameter in the Yang-Mills theory, that becomes visible only when it is realized in terms of the spin-charge separated variables.

Our result suggests the tantalizing possibility that long distance Einstein gravity metamorphoses into a renormalizable Yang-Mills theory at short distances.

Finally, we analyze the finite energy content of the spin-charge separated, static Yang-Mills theory using a Hamiltonian formulation. We find that closed knotted strings can also be supported by the $G(4,2)$ nonlinear $\sigma$-model, in a manner that involves the structure of (11). Furthermore, we propose that for finite energy configurations the space-time $\mathbb{R}^{4}$ becomes compactified into $\mathbb{S}^{3} \times \mathbb{R}^{1}$.

We conclude by presenting some physical interpretations of our results, together with suggestions on the directions for future research. 


\section{SOME NOTATION}

We consider a $S U(2)$ Yang-Mills gauge field $A=A_{a}^{i} \sigma^{i} d x^{a}$ in $\mathbb{R}^{4}(a, b, \ldots=1,2,3,4)$ with Euclidean signature. The Lie algebra generators coincide with the standard Pauli matrices $\sigma^{i}(i, j, \ldots=1,2,3)$ and we employ the complex combinations

$$
\sigma^{ \pm}=\frac{1}{2}\left(\sigma^{1} \pm i \sigma^{2}\right)
$$

For the gauge field this gives

$$
A=A_{a}^{i} \sigma^{i} d x^{a}=A_{a} \sigma^{3} d x^{a}+X_{a}^{+} \sigma^{-} d x^{a}+X_{a}^{-} \sigma^{+} d x^{a}
$$

where

$$
X_{a}^{ \pm}=A_{a}^{1} \pm i A_{a}^{2}
$$

The finite gauge transformation is

$$
A \rightarrow g A g^{-1}+2 i g d g^{-1}
$$

Note that in our notation the Yang-Mills coupling constant appears as a factor in front of the Lagrangian.

For an infinitesimal group element

$$
g=\exp \left\{\frac{i}{2} \boldsymbol{\epsilon} \cdot \boldsymbol{\sigma}\right\} \approx 1+\frac{i}{2} \hat{\epsilon}+\mathcal{O}\left(\epsilon^{2}\right)
$$

the gauge transformation takes the form

$$
\delta_{\hat{\epsilon}} A_{a}^{i}=\delta^{i j} \partial_{a} \epsilon^{j}+\varepsilon^{i j k} A_{a}^{k} \epsilon^{k}
$$

When the gauge transformation is in the direction of the Cartan subgroup $U_{C}(1) \in$ $S U(2)$

$$
g \sim h=e^{\frac{i}{2} \omega \sigma^{3}} \in U_{C}(1)
$$

the component $A_{a}^{3} \sim A_{a}$ transforms as a $U_{C}(1)$ gauge field

$$
\delta_{h} A_{a}=\partial_{a} \omega
$$


For the off-diagonal $X_{a}^{ \pm}$we get

$$
\delta_{h} X_{a}^{ \pm}=e^{\mp i \omega} X_{a}^{ \pm}
$$

Consequently, when we only consider gauge transformations in the direction of the Cartan subgroup, we can interpret the full $S U(2)$ gauge field as a charged $U_{C}(1)$ vector multiplet

$$
A_{a}^{i} \rightarrow\left(A_{a}, X_{a}^{ \pm}\right)
$$

Eventually we shall argue that even though we here introduce a particular (global) identification of the Cartan $U_{C}(1)$ in terms of the Pauli matrices, our results are independent of this particular choice. However, for the clarity of presentation we shall momentarily proceed with this choice of the Cartan direction in the $S U(2)$ Lie-algebra.

Finally, the Yang-Mills field strength tensor is

$$
F_{a b}^{i}=\partial_{a} A_{b}^{i}-\partial_{b} A_{a}^{i}+\epsilon^{i j k} A_{a}^{j} A_{b}^{k}
$$

In terms of the charged $U_{C}(1)$ vector multiplet it decomposes according to

$$
\begin{gathered}
F_{a b}^{3}=\partial_{a} A_{b}-\partial_{b} A_{a}+\frac{i}{2}\left(X_{a}^{+} X_{b}^{-}-X_{b}^{+} X_{a}^{-}\right)=F_{a b}+P_{a b} \\
F_{a b}^{ \pm}=F_{a b}^{1} \pm i F_{a b}^{2}=\left(\partial_{a} \pm i A_{a}\right) X_{b}^{ \pm}-\left(\partial_{b} \pm i A_{b}\right) X_{a}^{ \pm}=\mathrm{D}_{A a}^{ \pm} X_{b}^{ \pm}-\mathrm{D}_{A b}^{ \pm} X_{a}^{ \pm}
\end{gathered}
$$

Here the first term $F_{a b}$ in (11) is the $U_{C}(1)$ (Cartan) field strength tensor, and (12) involves the $U_{C}(1)$ covariant derivatives $\mathrm{D}_{A b}^{ \pm}$of the charged vector fields $X_{a}^{ \pm}$. These terms are clearly consistent with the $U_{C}(1)$ multiplet structure. The sole term that lacks an obvious physical interpretation in terms of the $U_{C}(1)$ multiplet, is the antisymmetric tensor $P_{a b}$ in (11). We now proceed to interpret it geometrically. 


\section{GRASSMANNIANS AND SPIN-CHARGE SEPARATION}

We shall now interpret the antisymmetric tensor $P_{a b}$ in (111). Explicitely we have

$$
P_{a b}=\frac{i}{2}\left(X_{a}^{+} X_{b}^{-}-X_{b}^{+} X_{a}^{-}\right)=A_{a}^{1} A_{b}^{2}-A_{b}^{1} A_{a}^{2}
$$

This is a real antisymmetric $4 \times 4$ matrix that obeys the quadratic relation

$$
P_{12} P_{34}-P_{13} P_{24}+P_{23} P_{14}=0
$$

In fact, using simple linear algebra one can show that any real $4 \times 4$ antisymmetric matrix $P_{a b}$ that is subject to the condition (14) can always be represented in the functional form (13) in terms of some two vectors $A_{a}^{1}$ and $A_{b}^{2}$. In projective geometry the relation (14) is known as the Klein quadric. It describes the embedding of the real Grassmannian $G(4,2)$ in the five dimensional projective space $\mathbb{R P}^{5}$ as a degree four hypersurface. This Grassmannian is the four dimensional manifold of two dimensional planes that are embedded in $\mathbb{R}^{4}$, and it can be identified with the homogeneous space

$$
G(4,2) \simeq \frac{S O(4)}{S O(2) \times S O(2)}
$$

It is convenient to describe the two dimensional planes in $\mathbb{R}^{4}$ in terms of a zweibein. For this we introduce an orthonormal doublet $e_{a}^{\alpha}(\alpha=1,2)$

$$
e_{a}^{\alpha} e_{a}^{\beta}=\delta^{\alpha \beta}
$$

that spans a generic two dimensional plane in $\mathbb{R}^{4}$. We can then represent the off-diagonal components of the gauge field as

$$
A_{a}^{\alpha}=M^{\alpha}{ }_{\beta} e_{a}^{\beta}
$$

where $M^{\alpha}{ }_{\beta}$ is a $2 \times 2$ matrix. In terms of the complex combination

$$
e_{a}=\frac{1}{\sqrt{2}}\left(e_{a}^{1}+i e_{a}^{2}\right)
$$


we have

$$
\begin{aligned}
& e_{a} e_{a}=0 \\
& e_{a} \bar{e}_{a}=1
\end{aligned}
$$

and we can write

$$
X_{a}^{+}=A_{a}^{1} \pm i A_{a}^{2}=\psi_{1} e_{a}+\psi_{2} \bar{e}_{a}
$$

Here the $\psi_{\alpha}$ are two arbitrary complex functions, they are linear combinations of the matrix elements of $M^{\alpha}{ }_{\beta}$.

When we substitute (16) into (13) we get

$$
P_{a b}=\frac{i}{2}\left(\left|\psi_{1}\right|^{2}-\left|\psi_{2}\right|^{2}\right) \cdot\left(e_{a} \bar{e}_{b}-e_{b} \bar{e}_{a}\right)=\frac{i}{2} \cdot \rho^{2} \cdot t_{3} \cdot\left(e_{a} \bar{e}_{b}-e_{b} \bar{e}_{a}\right)
$$

We have here introduced the three component unit vector

$$
\boldsymbol{t}=\frac{1}{\rho^{2}}\left(\begin{array}{ll}
\psi_{1}^{*} & \psi_{2}^{*}
\end{array}\right) \boldsymbol{\sigma}\left(\begin{array}{l}
\psi_{1} \\
\psi_{2}
\end{array}\right)=\left(\begin{array}{c}
\cos \phi \cdot \sin \theta \\
\sin \phi \cdot \sin \theta \\
\cos \theta
\end{array}\right)
$$

where we employ the following angular parametrization

$$
\begin{aligned}
& \psi_{1}=\rho e^{i \xi} \cos \frac{\theta}{2} e^{-i \phi / 2} \\
& \psi_{2}=\rho e^{i \xi} \sin \frac{\theta}{2} e^{i \phi / 2}
\end{aligned}
$$

The representation (17) has a motivation in terms of the properties of $P_{a b}$. For this we consider the action of the general linear group $G L(2, \mathbb{R})$ on the coordinates $A_{a}^{1}$ and $A_{a}^{2}$ that describe our generic two-plane

$$
\left(\begin{array}{l}
A_{a}^{1} \\
A_{a}^{2}
\end{array}\right) \stackrel{\mathrm{G}}{\longrightarrow}\left(\begin{array}{ll}
\alpha & \beta \\
\gamma & \delta
\end{array}\right)\left(\begin{array}{l}
A_{a}^{1} \\
A_{a}^{2}
\end{array}\right)
$$

Here

$$
\mathrm{G}=\left(\begin{array}{ll}
\alpha & \beta \\
\gamma & \delta
\end{array}\right)
$$


is the matrix realization of the $\mathrm{G} \in G L(2, \mathbb{R})$ on the two-dimensional plane in $\mathbb{R}^{4}$. This gives

$$
P_{a b} \stackrel{\mathrm{G}}{\longrightarrow}(\alpha \delta-\beta \gamma) P_{a b}=\operatorname{det} \mathrm{G} \cdot P_{a b}
$$

Thus $P_{a b}$ supports a one-dimensional representation of $G L(2, \mathbb{R})$, where the orbit is parametrized by the prefactor in (17)

$$
\left|\psi_{1}\right|^{2}-\left|\psi_{2}\right|^{2}=\rho^{2} \cdot t_{3}
$$

and the G-action corresponds to a scaling of the density $\rho$ according to

$$
\rho \stackrel{\mathrm{G}}{\longrightarrow} \sqrt{\operatorname{det} \mathrm{G}} \cdot \rho
$$

Clearly, the volume and orientation preserving subgroup $S L(2, \mathbb{R}) \in G L(2, \mathbb{R})$ is an invariance group of $P_{a b}$. The maximal compact subgroup $U_{I}(1) \in S L(2, \mathbb{R})$ is the internal invariance group of the decomposition (16). It acts on the fields according to

$$
\begin{aligned}
& e_{a} \rightarrow e^{-i \lambda} e_{a} \\
& \psi_{1} \rightarrow e^{i \lambda} \psi_{1} \\
& \psi_{2} \rightarrow e^{-i \lambda} \psi_{2}
\end{aligned}
$$

The vector $\boldsymbol{t}$ in (18) is invariant under the external $U_{C}(1)$ gauge transformation. The component $t_{3}$ is in addition invariant under the internal $U_{I}(1)$ gauge transformation. But the remaining two components transform nontrivially under the internal $U_{I}(1)$. With

$$
t_{ \pm}=t_{1} \pm i t_{2}
$$

the $U_{I}(1)$ transformation sends

$$
t_{ \pm}=\frac{1}{2}\left(t_{1} \pm i t_{2}\right) \rightarrow e^{\mp 2 i \lambda} t_{ \pm}
$$

where the factor of 2 reflects the fact that $\boldsymbol{t}$ is a bilinear in $\psi_{\alpha}$.

A priori, the r.h.s. of the decomposition (16) involves a total of nine independent field degrees of freedom. These are the four real components of the functions $\psi_{\alpha}$ and 
the five real components of the (complex) normalized vector $e_{a}$. But due to the internal $U_{I}(1)$ symmetry both sides of the decomposition (16) describe an equal number of eight independent field degrees of freedom. This coincides with the number of independent components in the off-diagonal gauge field and confirms that (16) yields a full, complete field decomposition of the off-diagonal components $X_{\mu}^{a}$ of the gauge field.

According to (21) the complex scalar fields $\psi_{1}$ and $\psi_{2}$ are oppositely charged with respect to the internal $U_{I}(1)$ gauge group. The vector field $e_{a}$ is also charged w.r.t. the internal $U_{I}(1)$ group while $A_{a}$ remains obviously intact under a $U_{I}(1)$ transformation. The obvious choice of a $U_{I}(1)$ connection is the composite vector field

$$
C_{a}=i \bar{e}_{b} \partial_{a} e_{b}
$$

as it transforms according to

$$
C_{a} \rightarrow C_{a}+\partial_{a} \lambda
$$

under the internal $U_{I}(1)$ transformation (21).

We note that in terms of the explicit parametrization (19), the $U_{I}(1)$ transformation (211) sends

$$
\phi \rightarrow \phi-2 \lambda
$$

We also note that the connection (23) admits a geometric interpretation as a spin connection that parallel transports the zweibein $\left(e_{a}^{1}, e_{a}^{2}\right)$.

According to (8), the Cartan subgroup $U_{C}(1)$ of the $S U(2)$ gauge group acts on the complex coefficients as follows,

$$
\psi_{1,2} \stackrel{h}{\longrightarrow} e^{-i \omega} \psi_{1,2}
$$

or in terms of the parametrization (19)

$$
\xi \rightarrow \xi-\omega
$$

while $e_{a}$ remains intact. Consequently we can interpret the $S U(2)$ gauge field as the $U_{C}(1)$ multiplet

$$
A_{a}^{i} \sim\left(A_{a}, \psi_{1}, \psi_{2}, e_{a}\right)
$$


where $A_{a}$ is the $U_{C}(1)$ gauge field, the complex scalar fields $\psi_{\alpha}$ are equally charged w.r.t. the $U_{C}(1)$, and the complex vector field $e_{a}$ is $U_{C}(1)$ neutral.

We can also interpret the gauge field as the $U_{I}(1)$ multiplet

$$
A_{a}^{i} \sim\left(C_{a}, \psi_{1}, \psi_{2}, A_{a}\right)
$$

where $C_{a}$ is the $U_{I}(1)$ gauge field, the complex scalar fields are oppositely charged w.r.t. the $U_{I}(1)$ and the vector field $A_{a}$ is $U_{I}(1)$ neutral.

Notice that the $\psi_{\alpha}$ are scalars and $e_{a}$ is a vector under $S O(4)$ rotations (a.k.a. Lorentz transformations) in $\mathbb{R}^{4}$. Since $e_{a}$ is neutral under the $U_{C}(1) \in S U(2)$ gauge group while the $\psi_{\alpha}$ transform nontrivially, we conclude that the decomposition (16) entails a separation between the spin and the charge in the off-diagonal components $X_{a}^{ \pm}$of the $S U(2)$ gauge field. The spinless scalar fields $\psi_{\alpha}$ describe the $U_{C}(1)$ charge degrees of freedom of the $X_{a}^{ \pm}$, and the $U_{C}(1)$ neutral vector field $e_{a}$ describes their spin degree of freedom.

The present separation between the spin and the charge degrees of freedom in $X_{a}^{ \pm}$is quite analogous to the slave-boson decomposition of an (nonrelativistic) electron, widely employed in attempts to explain high-temperature superconductivity [14]. There, the spin-charge separation is introduced as an alternative to the BCS superconductivity. Instead of the Higgs effect in terms of the Cooper pairs, superconductivity emerges when the analog of our variable $\rho$ forms a condensate,

$$
<v a c|\rho| v a c>=\Delta \neq 0
$$




\section{ELECTRIC AND MAGNETIC COMPONENTS}

Consider the tensor part of $P_{a b}$ in (17),

$$
H_{a b}=\frac{i}{2}\left(e_{a} \bar{e}_{b}-e_{b} \bar{e}_{a}\right)
$$

We define its "electric" $\left(p_{i}\right)$ and "magnetic" $\left(q_{i}\right)$ components in the usual manner, by setting

$$
\begin{aligned}
& p_{i}=H_{0 i}=\frac{i}{2}\left(e_{0} e_{i}^{*}-e_{i} e_{0}^{*}\right) \\
& q_{i}=\frac{1}{2} \epsilon_{i j k} H_{j k}=\frac{i}{2} \epsilon_{i j k} e_{j} e_{k}^{*}
\end{aligned}
$$

These two vectors are subject to the orthogonality relations

$$
\begin{gathered}
\boldsymbol{p} \cdot \boldsymbol{q}=0 \\
\boldsymbol{p} \cdot \boldsymbol{p}+\boldsymbol{q} \cdot \boldsymbol{q}=\frac{1}{4}
\end{gathered}
$$

Together with the Poynting vector

$$
s=p \times q
$$

we then have an orthogonal triplet in $\mathbb{R}^{3}$.

The relation (26) can also be inverted, the result is

$$
\boldsymbol{e}=\left(\begin{array}{c}
e_{0} \\
e_{1} \\
e_{2} \\
e_{3}
\end{array}\right)=e^{i \eta}\left(\begin{array}{c}
\sqrt{2}|\boldsymbol{p}| \\
\frac{2 \boldsymbol{s}+i \boldsymbol{p}}{\sqrt{2}|\boldsymbol{p}|}
\end{array}\right) \equiv e^{i \eta} \hat{\boldsymbol{e}}
$$

Here $\eta$ is an overall phase of $\boldsymbol{e}$. This phase is invisible to $\boldsymbol{p}$ and $\boldsymbol{q}$ since it does not contribute to the bilinear combination (25). But this phase is subject to the internal $U_{I}(1)$ gauge transformation (21) which sends

$$
\eta \rightarrow \eta-\lambda
$$

Thus the phase transformation (29) determines a rotation between the real and imaginary components $e_{a}^{1}$ and $e_{a}^{2}$ of the complex vector $e_{a}$ in $\mathbb{R}^{4}$. 
Now consider the action of $S O(4)$ rotations (a.k.a. Lorentz transformations) on $e_{a}$. We are particularly interested in the effect of an infinitesimal $S O(4)$ (Lorentz) boost in a generic spatial direction $\varepsilon_{i}$. As a component of a four-vector, the $e_{a}$ should transform in the following manner

$$
\begin{aligned}
& \Lambda_{\varepsilon} e_{0}=-\varepsilon_{i} e_{i} \\
& \Lambda_{\varepsilon} e_{i}=-\varepsilon_{i} e_{0}
\end{aligned}
$$

This is clearly a $S O(4)$ rotation, it preserves the orthonormality relations (15).

On the other hand, we expect that when we realize this boost transformation on the electric and magnetic components (26) we should get the familiar results

$$
\begin{aligned}
& \delta_{\varepsilon} \boldsymbol{p}=\boldsymbol{q} \times \boldsymbol{\varepsilon} \\
& \delta_{\varepsilon} \boldsymbol{q}=\boldsymbol{p} \times \boldsymbol{\varepsilon}
\end{aligned}
$$

Curiously, we find that when we substitute this in the explicit realization (28) there is a difference between (30) and (31): If we compare the action of $\Lambda_{\varepsilon}$ in (30) with the action of $\delta_{\varepsilon}$ on $e_{a}$ which is defined using (31) and (28), the two descriptions of the boost differ from each other by the (infinitesimal) phase

$$
\left(\Lambda_{\varepsilon}-\delta_{\varepsilon}\right) e_{a}=-i \Theta(\boldsymbol{p}, \boldsymbol{q} ; \boldsymbol{\varepsilon}) \cdot e_{a}
$$

As a consequence the difference between the two Lorentz boosts is a (infinitesimal) shift in the angle $\eta$ in (28) according to

$$
\eta \rightarrow \eta-\Theta(\boldsymbol{p}, \boldsymbol{q} ; \boldsymbol{\varepsilon})
$$

This is an infinitesimal internal $U_{I}(1)$ gauge rotation (29).

Explicitely, we have

$$
\Theta(\boldsymbol{p}, \boldsymbol{q} ; \boldsymbol{\varepsilon})=\frac{\boldsymbol{p} \cdot \boldsymbol{\varepsilon}}{|\boldsymbol{p}|^{2}}=\varepsilon^{i} \frac{\partial}{\partial p_{i}} \ln |\boldsymbol{p}|
$$

One can verify that this quantity obeys the one-cocycle condition

$$
\delta_{\varepsilon_{1}} \Theta\left(\boldsymbol{p}, \boldsymbol{q} ; \boldsymbol{\varepsilon}_{2}\right)-\delta_{\varepsilon_{2}} \Theta\left(\boldsymbol{p}, \boldsymbol{q} ; \boldsymbol{\varepsilon}_{1}\right)=0
$$


and as a consequence (32) is a one-cocycle.

The result means that the action of the boost $\delta_{\varepsilon}$ on the vector field $e_{a}$ determines a projective representation of the (Euclidean) Lorentz group. In particular, the vector field $\hat{e}_{a}$ in (28)

$$
\hat{\mathbf{e}}=e^{-i \eta} \mathbf{e}
$$

transforms under the projective representation according to

$$
\delta_{\varepsilon} \hat{\mathbf{e}}=\Lambda_{\varepsilon} \hat{\mathbf{e}}+i \Theta \hat{\mathbf{e}}
$$

We conclude that the phase $\eta$ in (28) is a non-trivial field degree of freedom. If we set $\eta=0$ in (28), a spatial boost will generate a nontrivial $\eta$ determined by the one-cocycle (32). Moreover, since the cocycle depends only on the electric component of (25) we propose that $\eta$ can be viewed as a phase (angular) variable for electric circulation.

Consider the internal $U_{I}(1)$ connection (23) . It admits the following explicit realization

$$
C_{a}=i \overline{\mathbf{e}} \cdot \partial_{a} \boldsymbol{e}=i \hat{\overline{\mathbf{e}}} \cdot \partial_{a} \hat{\mathbf{e}}-\partial_{a} \eta=\mathbf{2}|\boldsymbol{q}|\left(\boldsymbol{k} \times \boldsymbol{l} \cdot \partial_{a} \boldsymbol{k}\right)-\partial_{a} \eta=\hat{C}_{a}-\partial_{a} \eta
$$

Here $\boldsymbol{k}$ and $\boldsymbol{l}$ are two mutually orthogonal unit vectors in the electric and magnetic directions respectively,

$$
\begin{aligned}
& \boldsymbol{p}=|\boldsymbol{p}| \boldsymbol{k}=\frac{1}{2 \sqrt{2}} \cos \vartheta \cdot \boldsymbol{k} \\
& \boldsymbol{q}=|\boldsymbol{q}| \boldsymbol{l}=\frac{1}{2 \sqrt{2}} \sin \vartheta \cdot \boldsymbol{l}
\end{aligned}
$$

Since both $\boldsymbol{k}$ and $\boldsymbol{l}$ remain intact under both the external $U_{C}(1)$ and the internal $U_{I}(1)$ gauge transformations we conclude that the vector field

$$
\hat{C}_{a}=C_{a}+\partial_{a} \eta=i \hat{\overline{\mathbf{e}}} \cdot \partial_{a} \hat{\mathbf{e}}=2|\boldsymbol{q}|\left(\boldsymbol{k} \times \boldsymbol{l} \cdot \partial_{a} \boldsymbol{k}\right)=\frac{2 \boldsymbol{p} \cdot \partial_{a} \boldsymbol{s}}{\boldsymbol{p}^{2}}
$$

is gauge invariant both under the external $U_{C}(1)$ and under the internal $U_{I}(1)$ gauge transformations; The internal $U_{I}(1)$ acts only on the phase variable $\eta$ in (33) according to (29). 
If we introduce the normalization

$$
\omega_{a}=-\frac{1}{2|\boldsymbol{q}|} \hat{C}_{a}=-2 \boldsymbol{k} \times \boldsymbol{l} \cdot \partial_{a} \boldsymbol{k}
$$

we arrive at

$$
\partial_{a} \omega_{b}-\partial_{b} \omega_{a}=\boldsymbol{l} \cdot \partial_{a} \boldsymbol{l} \times \partial_{b} \boldsymbol{l}
$$

This is the pull-back of the volume two-form on $\mathbb{S}^{2}$ that also appears in the second term of (11). As a consequence (36) admits the geometric interpretation as the Kirillov one-form for the co-adjoint orbit $\mathbb{S}^{2}=S U(2) / U(1)$, in the magnetic direction of $\boldsymbol{l}$.

The result (37) follows directly from the formal properties of the unit vectors $\boldsymbol{k}$ and $\boldsymbol{l}$. Alternatively, it can be verified by using an explicit angular representation of these vectors. If we denote by $\boldsymbol{m}$ the unit vector in the direction of the Poynting vector,

$$
\boldsymbol{m}=-\boldsymbol{k} \times \boldsymbol{l}
$$

we can represent the orthonormal triplet $(\boldsymbol{k}, \boldsymbol{l}, \boldsymbol{m})$ as follows,

$$
\begin{aligned}
\boldsymbol{k} & =\cos \gamma \cdot \boldsymbol{u}_{x}+\sin \gamma \cdot \boldsymbol{u}_{y} \\
\boldsymbol{l} & =\boldsymbol{u}_{z} \\
\boldsymbol{m} & =-\sin \gamma \cdot \boldsymbol{u}_{x}+\cos \gamma \cdot \boldsymbol{u}_{y}
\end{aligned}
$$

where

$$
\boldsymbol{u}_{x}=\left(\begin{array}{c}
\cos \alpha \cos \beta \\
\sin \alpha \cos \beta \\
-\sin \beta
\end{array}\right) \quad \& \quad \boldsymbol{u}_{y}=\left(\begin{array}{c}
-\sin \alpha \\
\cos \alpha \\
0
\end{array}\right) \quad \& \quad \boldsymbol{u}_{z}=\left(\begin{array}{c}
\cos \alpha \sin \beta \\
\sin \alpha \sin \beta \\
\cos \beta
\end{array}\right)
$$

We then have

$$
A_{M}=-\frac{1}{4} \omega=-\frac{1}{2}(\cos \beta \cdot d \alpha+d \gamma)
$$

and

$$
F_{M}=-\frac{1}{4} d \omega=-\frac{1}{2} \sin \beta \cdot d \alpha \wedge d \beta
$$

In (40) we recognize the connection one-form $A_{M}$ and in (41) the curvature two-form $F_{M}$ (magnetic field) of the Dirac magnetic monopole. 
For (35) we get from (34)

$$
\hat{C}=-\sqrt{2} \sin \vartheta \cdot[\cos \beta \cdot d \alpha+d \gamma]
$$

This vanishes when we go to the purely electric limit $\vartheta=0$, and coincides with (twice) the Dirac monopole connection (40) when $\vartheta=\pi / 4$ and the strength of the electric and magnetic fields are equal. 


\section{A HIGGS EFFECT}

The three components of the unit vector $\boldsymbol{t}$ that we have introduced in (18) are bilinear in the complex functions $\psi_{1}$ and $\psi_{2}$. Since these functions are $S O(4)$ a.k.a. Lorentz scalars, the unit vector $\boldsymbol{t}$ is also a Lorentz scalar. But its components $t_{ \pm}$are not invariant under the internal $U_{I}(1)$ gauge transformations.

If instead we introduce a new three component unit vector $\boldsymbol{n}$ such that

$$
\begin{aligned}
& n_{ \pm}=e^{2 i \eta} t_{ \pm} \\
& n_{3}=t_{3}
\end{aligned}
$$

then this new unit vector is invariant under both the external $U_{C}(1)$ and the internal $U_{I}(1)$ gauge transformations. Explicitely, in terms of the angular variables in (18) we have

$$
\boldsymbol{n}=\left(\begin{array}{c}
\cos (\phi+2 \eta) \cdot \sin \theta \\
\sin (\phi+2 \eta) \cdot \sin \theta \\
\cos \theta
\end{array}\right)
$$

Here

$$
\phi+2 \eta
$$

is a $U_{C}(1) \times U_{I}(1)$ invariant combination of the $U_{I}(1)$ dependent variables $\phi$ and $\eta$.

In the sequel we shall propose that $\hat{\mathbf{e}}_{a}$ in (28), $\hat{C}_{a}$ in (35) and $\boldsymbol{n}$ are the obvious $U_{C}(1) \times U_{I}(1)$ gauge invariant variables for describing the Yang-Mills theory. For this we note that the relation (35) between $C_{a}$ and $\hat{C}_{a}$ is a version of the familiar (linear) Higgs relation between the $U(1)$ gauge vector field and the gauge invariant (massive) vector field in the standard abelian Higgs model:

In the case of conventional Higgs mechanism, a $U(1)$ gauge field combines with the gradient of the phase of a complex scalar field into a gauge invariant vector field. The modulus of the complex scalar field remains as an additional, independent gauge invariant field variable. When this modulus develops a nonvanishing ground state expectation value the gauge invariant vector field becomes massive. 
Here, the $U_{I}(1)$ gauge field $C_{a}$ combines similarly with the phase $\eta$ of the complex vector field $\mathbf{e}_{a}$ into the gauge invariant vector field $\hat{C}_{a}$. This leaves the vector field $\hat{\mathbf{e}}_{a}$ in (28) as an additional independent and $U_{I}(1)$ invariant field variable. Furthermore, the transition from $\boldsymbol{t}$ to the $U_{I}(1)$ invariant $\boldsymbol{n}$ can be viewed as a nonlinear version of the Higgs mechanism. Note that all of these three $U_{I}(1)$ invariant field variables are also invariant under the $U_{C}(1)$ gauge transformations.

We remind that due to the presence of the one-cocycle (32) the vector fields $\hat{C}_{a}$ and $\hat{\mathbf{e}}_{a}$ are not $S O(4)$ vectors. Nor are the \pm -components of $\boldsymbol{n}$ scalars under $S O(4)$. Instead, all of these $U_{I}(1)$ gauge invariant quantities transform under a projective representation of the spatial $S O(4)$ (a.k.a. Lorentz) group.

The breaking of the Lorentz invariance by the one-cocycle has important physical consequences. For this we note that the two components $A_{a}^{1}$ and $A_{a}^{2}$ of the $S U(2)$ gauge field appear symmetrically in the Yang-Mills Lagrangian, they can be exchanged by a global gauge transformation. Consequently we can expect that in terms of the spincharge separated variables the Lagrangian should display a similar global symmetry between the two complex scalar fields $\psi_{1}$ and $\psi_{2}$. This symmetry should translate into a global $O(3)$ rotation invariance when represented in terms of the unit vector $\boldsymbol{n}$ in (43); See the first two terms in (11). But the presence of such a global $O(3)$ symmetry poses a problem. When we select a ground state direction of $\boldsymbol{n}$ we break the global $O(3)$ invariance explicitly, in a manner which in general leads to two a priori massless Goldstone bosons.

The violation of Lorentz invariance by the one-cocycle appears to remove the Goldstone bosons: Since we have no reason to expect that the ground state of the theory violates Lorentz invariance and since $n_{3}$ is the sole Lorentz invariant component of $\boldsymbol{n}$, the only possible Lorentz invariant ground state direction for $\boldsymbol{n}$ is

$$
\boldsymbol{n} \rightarrow \pm\left(\begin{array}{l}
0 \\
0 \\
1
\end{array}\right) \equiv \hat{z}
$$


Any nonvanishing ground state value for the components $n_{ \pm}$breaks Lorentz invariance due to the presence of the one-cocycle.

In particular, we conclude that at large spatial distances the unit vector $\boldsymbol{n}$ should become asymptotically parallel with the $z$-axis,

$$
\boldsymbol{n} \stackrel{|x| \rightarrow \infty}{\longrightarrow} \pm \hat{z}
$$

Alternatively, in terms of the vector $\boldsymbol{t}$ the only $U_{I}(1)$ gauge invariant asymptotic ground state direction is

$$
t \rightarrow \pm \hat{z}
$$

since any other asymptotic direction violates the internal $U_{I}(1)$ gauge invariance. 


\section{YANG-MILLS IN GAUGE INVARIANT SPIN-CHARGE VARIABLES}

We now proceed to inspect how the separation between the spin and the charge manifests itself in the Yang-Mills Lagrangian. We start by recalling the tree-level gauge fixed Euclidean space Yang-Mills Lagrangian

$$
L_{Y M}=\frac{1}{4}\left(F_{a b}^{i}\right)^{2}+\frac{\xi}{2}\left|\mathrm{D}_{A a}^{+} X_{a}^{+}\right|^{2}+L_{\text {ghost }}
$$

Note that we have here introduced a gauge fixing term only for the off-diagonal components $X_{a}^{ \pm}$of the gauge field. For reasons that will eventually become transparent, we do not introduce any gauge fixing term in the direction of the abelian subgroup $U_{C}(1) \in S U(2)$. The last term $L_{\text {ghost }}$ denotes the ghost contribution. In the sequel its explicit form will not be of importance to us. We only need to observe that it is entirely independent of the gauge fixing parameter $\xi$ [19], [20].

In our approach we do not introduce decomposed variables in the path integral. That would only lead to unnecessary complications. Instead we propose that the appropriate stage to implement the spin-charge separation is at the level of the effective Yang-Mills action which has been computed in the covariant background field formalism. This effective action accounts for all quantum fluctuations in the gauge field. But since its explicit form is not available beyond a few leading terms in a loop expansion, we need to resort to an indirect analysis.

By general arguments of gauge invariance we can expect that the full effective action is a functional of the background field strength tensor $F_{a b}^{i}$ and its background covariant derivatives. In the low momentum infrared limit we can ignore the derivative contributions, hence in this limit the effective action involves only the field strength tensor. Since the full result is unknown to us, for simplicity we proceed by considering the infrared limit only in its lowest order. This limit coincides with the classical Lagrangian (45), but excluding the ghost contribution. Consequently our starting point will be the classical Yang-Mills Lagrangian (45). Indeed, the classical Lagrangian should be an important ingredient of the full quantum action. We now proceed to subject it to the separation 
between the spin and the charge.

When we introduce (11) and (12) we find for the classical Yang-Mills Lagrangian

$$
\begin{gathered}
L_{Y M}=\frac{1}{4}\left(F_{a b}^{i}\right)^{2}+\frac{\xi}{2}\left|\mathrm{D}_{A a}^{+} X_{a}^{+}\right|^{2} \\
=\frac{1}{4}\left(F_{a b}+2 \rho^{2} n_{3} H_{a b}\right)^{2}+\frac{1}{2}\left|\mathrm{D}_{A a}^{+} X_{b}^{+}\right|^{2}+\frac{3}{8}\left(1-n_{3}^{2}\right) \rho^{4}-\frac{3}{8} \rho^{4}+\frac{\xi-1}{2}\left|\mathrm{D}_{A a}^{+} X_{a}^{+}\right|^{2}
\end{gathered}
$$

The reason why we present the third and fourth terms in (46) in this particular manner becomes evident as we proceed.

Note that we have here overlooked a surface contribution that originates from the difference

$$
\mathrm{D}_{A a}^{+} X_{b}^{+} \mathrm{D}_{A b}^{-} X_{a}^{-}-\mathrm{D}_{A a}^{+} X_{a}^{+} \mathrm{D}_{A b}^{-} X_{b}^{-}
$$

Explicitely, the surface contribution is

$$
\partial_{a}\left\{\frac{1}{2}\left[X_{a}^{+} \mathrm{D}_{A b}^{-} X_{b}^{-}+X_{a}^{-} \mathrm{D}_{A b}^{+} X_{b}^{+}\right]-\frac{1}{2} \partial_{b}\left(X_{a}^{+} X_{b}^{-}\right)\right\}
$$

We first observe that in (46) there are two particularly interesting values for the gauge fixing parameter $\xi$. These are the value $\xi=1$, and the limit $\xi \rightarrow \infty$.

If we select $\xi=1$ the last term in (46) becomes absent. In particular, when $\xi=1$ there are no terms present in the Lagrangian barring the surface term, where the Lorentz index in the off-diagonal components $X_{a}^{ \pm}$becomes contracted with the Lorentz indices that are carried by the other quantities, such as the derivative operator $\partial_{a}$. For $\xi=1$ the Lorentz indices in $X_{a}^{ \pm}$are only contracted internally between different contributions of the $X_{a}^{ \pm}$.

Since the ghost Lagrangian is independent of $\xi$, by arguments of gauge invariance we expect that this property persists to all orders of perturbation theory. In particular we expect that in the full $\xi=1$ quantum effective action the Lorentz indices carried by the (background) fields $X_{a}^{ \pm}$are only contracted internally, between different contributions of $X_{a}^{ \pm}$. This indicates that we can (crudely) analyze the feasibility of the spin-charge 
separation by considering the Lagrangian (46), with $\xi=1$ and ignoring the ghost contributions. As a consequence we limit our interest to only the following four terms,

$$
L_{Y M}=\frac{1}{4}\left(F_{a b}+2 \rho^{2} n_{3} H_{a b}\right)^{2}+\frac{1}{2}\left|\mathrm{D}_{A a}^{+} X_{b}^{+}\right|^{2}+\frac{3}{8}\left(1-n_{3}^{2}\right) \rho^{4}-\frac{3}{8} \rho^{4}
$$

Similar conclusions can be drawn in the gauge that emerges when we send $\xi \rightarrow \infty$. In this limit we obtain in addition the maximal abelian gauge (MAG) condition

$$
\left(\partial_{a} \pm i A_{a}\right) X_{a}^{ \pm}=\mathrm{D}_{A a}^{ \pm} X_{a}^{ \pm}=0
$$

It is known [19], 21] that this gauge condition is also the (Euler-Lagrange) variational equation that describes the gauge orbit extrema of the following quantity,

$$
\mathcal{R}=\int d^{4} x X_{a}^{+} X_{a}^{-}
$$

In particular,

$$
\rho^{2}=\left|\psi_{1}\right|^{2}+\left|\psi_{2}\right|^{2}=X_{a}^{+} X_{a}^{-}
$$

where $\rho$ is the density that we have introduced in (19). Notice that with (49) the first two terms in the surface contribution (47) vanish.

The variable $\rho$ has an interpretation as a condensate; see (24). In the context of the maximal abelian gauge this interpretation of $\rho$ has been discussed widely in the literature [19], [21]: The extrema values of $\rho$ on the gauge orbit are obviously gauge invariant and according to (150) correspond to gauge field configurations that are subject to the MAG gauge condition (49) and we refer to [19], 21] for further discussion.

From (20) we also conclude that selecting the extrema value of $\rho$ breaks the Grassmannian $G L(2, \mathbb{R})$ into $S L(2, \mathbb{R})$.

We now proceed to analyze the Lagrangian (48). In the present section our goal will be to represent it in terms of the $U_{C}(1) \times U_{I}(1)$ gauge invariant variables. We shall find that this can be achieved by a change of variables, with no need to any additional explicit gauge fixing. This will justify a posteriori why in (45) we have introduced the gauge fixing term only for the off-diagonal $X_{a}^{ \pm}$. 
We first consider the second term in (48). Using (18) we can write this term as

$$
\left|\mathrm{D}_{A a}^{+} X_{b}^{+}\right|^{2}=\left|\mathrm{D}_{A a}^{C} \psi_{1}\right|^{2}+\left|\mathrm{D}_{A a}^{C} \psi_{2}\right|^{2}+\rho^{2}\left|\mathrm{D}_{A a}^{C} e_{b}\right|^{2}+\frac{1}{2} \rho^{2} t_{+}\left(\overline{\mathrm{D}}_{A_{a}}^{C} \bar{e}_{b}\right)^{2}+\frac{1}{2} \rho^{2} t_{-}\left(\mathrm{D}_{A a}^{C} e_{b}\right)^{2}
$$

Here $\mathrm{D}_{A a}^{C}$ is the following $U_{C}(1) \times U_{I}(1)$ covariant derivative,

$$
\begin{aligned}
& \mathrm{D}_{A a}^{C} \psi_{1}=\left(\partial_{a}+i A_{a}-i C_{a}\right) \psi_{1} \\
& \mathrm{D}_{A a}^{C} \psi_{2}=\left(\partial_{a}+i A_{a}+i C_{a}\right) \psi_{2} \\
& \mathrm{D}_{A a}^{C} e_{b}=\left(\partial_{a}+i C_{a}\right) e_{b}
\end{aligned}
$$

Note that even though the $t_{ \pm}$are not invariant under the internal $U_{I}(1)$ gauge transformations (22), since $\mathrm{D}_{A a}^{C} e_{b}$ transforms according to

$$
\mathrm{D}_{A a}^{C} e_{b} \rightarrow e^{-i \lambda} \mathrm{D}_{A a}^{C} e_{b}
$$

the Lagrangian (51) is gauge invariant under both $U_{I}(1)$ and $U_{C}(1)$ gauge transformations.

We introduce the $U_{C}(1) \times U_{I}(1)$ invariant supercurrent [4], 22]

$$
J_{a}=\frac{i}{2 \rho^{2}}\left\{\psi_{1}^{*} \mathrm{D}_{A a}^{C} \psi_{1}-\psi_{1} \overline{\mathrm{D}}_{A a}^{C} \psi_{1}^{*}+\psi_{2}^{*} \mathrm{D}_{A a}^{C} \psi_{2}-\psi_{2} \overline{\mathrm{D}}_{A a}^{C} \psi_{2}^{*}\right\}
$$

From this we can solve for $A_{a}$ in favor of $J_{a}$. The result is

$$
A_{a}=-J_{a}+\frac{i}{2 \rho^{2}}\left\{\psi_{1}^{*} \overleftrightarrow{\partial_{a}} \psi_{1}+\psi_{2}^{*} \overleftrightarrow{\partial_{a}} \psi_{2}\right\}+n_{3} \cdot C_{a}
$$

When we substitute this in (51) we get for the first two terms

$$
\left|\mathrm{D}_{A a}^{C} \psi_{1}\right|^{2}+\left|\mathrm{D}_{A a}^{C} \psi_{2}\right|^{2}=\left(\partial_{\mu} \rho\right)^{2}+\frac{1}{4} \rho^{2}\left(\mathrm{D}_{a}^{\hat{C}} \boldsymbol{n}\right)^{2}+\rho^{2} J_{a}^{2}
$$

Here we have defined the covariant derivative operator

$$
\left(\mathrm{D}_{a}^{\hat{C}}\right)^{i j}=\delta^{i j} \partial_{a}+2 \epsilon^{i j 3} \hat{C}_{a} \quad(i, j=1,2,3)
$$

Note in particular that the middle term in the r.h.s. of (52) is Lorentz invariant even though both the components $n_{ \pm}$and the connection $\hat{C}_{a}$ transform according to a projective representation of $S O(4)$. The covariant derivative (53) compensates for the lack of $S O(4)$ invariance (a.k.a. Lorentz invariance) in the $i=1,2$ components of $\boldsymbol{n}$. 
With (52) we have achieved our goal, in the sense that the r.h.s. of (52) involves only quantities which are $U_{C}(1) \times U_{I}(1)$ invariant.

We now proceed to the third term in (51). For this we get

$$
\begin{gathered}
\rho^{2}\left|\left(\partial_{a}+i C_{a}\right) \boldsymbol{e}\right|^{2}=\rho^{2}\left|\left(\partial_{a}+i \hat{C}_{a}\right) \hat{\boldsymbol{e}}\right|^{2}=\frac{\rho^{2}}{2}\left\{\left(\partial_{a} \boldsymbol{p}\right)^{2}+\left(\partial_{a} \boldsymbol{q}\right)^{2}\right\} \\
=\frac{\rho^{2}}{16}\left\{\cos ^{2} \vartheta \cdot\left(\partial_{a} \boldsymbol{k}\right)^{2}+\sin ^{2} \vartheta \cdot\left(\partial_{a} \boldsymbol{l}\right)^{2}+\left(\partial_{a} \vartheta\right)^{2}\right\}
\end{gathered}
$$

Clearly, this involves only manifestly $U_{C}(1) \times U_{I}(1)$ invariant quantities.

We observe that there is the following apparent structural similarity between a contribution to the second and third terms in the r.h.s. of (152), and the r.h.s. of (54),

$$
\begin{array}{cccc}
\left(\partial_{a} \boldsymbol{n}\right)^{2} & \leftrightarrow & \cos ^{2} \vartheta \cdot\left(\partial_{a} \boldsymbol{k}\right)^{2}+\sin ^{2} \vartheta \cdot\left(\partial_{a} \boldsymbol{l}\right)^{2} \\
J_{a}^{2} & \leftrightarrow & \left(\partial_{a} \vartheta\right)^{2}
\end{array}
$$

In 4] it has been suggested that this structural similarity can be interpreted in terms of an electric-magnetic duality. Here we propose that it suggests a duality between the spin and the charge.

The last two terms in (51) can also be represented in terms of $U_{C}(1) \times U_{I}(1)$ gauge invariant variables as follows,

$$
\begin{gathered}
\frac{1}{2} \rho^{2} t_{+}\left(\mathrm{D}_{A a}^{C} \bar{e}_{b}\right)^{2}=\frac{1}{2} \rho^{2} n_{+}\left(\partial_{a} \hat{\bar{e}}_{b}\right)^{2} \\
=\frac{1}{128} \frac{\rho^{2} n_{+}}{|\boldsymbol{s}|^{2}}<\partial_{a}(\boldsymbol{p}+\boldsymbol{q}), \boldsymbol{p}-\boldsymbol{q}-4 \sqrt{2} i \boldsymbol{s}><\partial_{a}(\boldsymbol{p}-\boldsymbol{q}), \boldsymbol{p}+\boldsymbol{q}-4 \sqrt{2} i \boldsymbol{s}> \\
\frac{1}{2} \rho^{2} t_{-}\left(\mathrm{D}_{A a}^{C} e_{b}\right)^{2}=\frac{1}{2} \rho^{2} n_{-}\left(\partial_{a} \hat{e}_{b}\right)^{2}= \\
=\frac{1}{128} \frac{\rho^{2} n_{-}}{|\boldsymbol{s}|^{2}}<\partial_{a}(\boldsymbol{p}+\boldsymbol{q}), \boldsymbol{p}-\boldsymbol{q}+4 \sqrt{2} i \boldsymbol{s}><\partial_{a}(\boldsymbol{p}-\boldsymbol{q}), \boldsymbol{p}+\boldsymbol{q}+4 \sqrt{2} i \boldsymbol{s}>
\end{gathered}
$$


Shortly we shall argue that these two terms admit a geometrical interpretation in the Grassmannian framework. However, prior to this we consider the remaining contributions to the Yang-Mills Lagrangian.

We proceed with the first term in (51). When we eliminate $A_{a}$ in favor of the supercurrent $J_{a}$ we get for this term

$$
\frac{1}{4}\left(F_{a b}+2 \rho^{2} t_{3} H_{a b}\right)^{2}=\frac{1}{4}\left(L_{a b}+M_{a b}-n_{3} K_{a b}-2 \rho^{2} n_{3} H_{a b}\right)^{2}
$$

Here

$$
\begin{aligned}
L_{a b} & =\partial_{a} J_{b}-\partial_{b} J_{a} \\
M_{a b} & =\frac{1}{2} \boldsymbol{n} \cdot \mathrm{D}_{a}^{\hat{C}} \boldsymbol{n} \times \mathrm{D}_{b}^{\hat{C}} \boldsymbol{n} \\
K_{a b} & =\partial_{a} \hat{C}_{b}-\partial_{b} \hat{C}_{a}
\end{aligned}
$$

In particular, (57) and (58) involve only quantities which are explicitely $U_{C}(1)$ and $U_{I}(1)$ invariant. The covariant derivative (53) ensures that all quantities are also independently $S O(4)$ (Lorentz) invariant.

We note that we can write the second and third terms in the r.h.s. of (157) as follows,

$$
n_{3}\left(\partial_{a} \hat{C}_{b}-\partial_{b} \hat{C}_{a}\right)-\frac{1}{2} \boldsymbol{n} \cdot \mathrm{D}_{a}^{\hat{C}} \boldsymbol{n} \times \mathrm{D}_{b}^{\hat{C}} \boldsymbol{n}=\partial_{a}\left[n_{3} \hat{C}_{b}\right]-\partial_{b}\left[n_{3} \hat{C}_{a}\right]-\frac{1}{2} \boldsymbol{n} \cdot \partial_{a} \boldsymbol{n} \times \partial_{b} \boldsymbol{n}
$$

The structure in (59) is reminiscent of the 't Hooft tensor [23]. The last term is the pull-back of the volume two-form on $\mathbb{S}^{2}$, and if we introduce the corresponding Kirillov one-form (36)

$$
-\frac{1}{2} \boldsymbol{n} \cdot \partial_{a} \boldsymbol{n} \times \partial_{b} \boldsymbol{n}=\partial_{a} Q_{b}-\partial_{b} Q_{a}
$$

we can combine the first three terms in the r.h.s. of (57) into

$$
L_{a b}+M_{a b}-n_{3} K_{a b}=\partial_{a}\left(J_{b}-n_{3} \hat{C}_{b}-Q_{b}\right)-\partial_{b}\left(J_{a}-n_{3} \hat{C}_{a}-Q_{a}\right)
$$

In summary, when we combine our results we find that in terms of the spin-charge separated variables the Yang-Mills Lagrangian has the following $U_{C}(1) \times U_{I}(1)$ invariant 
form

$$
\begin{aligned}
L_{Y M}=\frac{1}{4} \mathcal{F}_{a b}^{2}+ & \frac{1}{2}\left(\partial_{a} \rho\right)^{2}+\frac{1}{2} \rho^{2} J_{a}^{2}+\frac{1}{8} \rho^{2}\left(\mathrm{D}_{a}^{\hat{C}} \boldsymbol{n}\right)^{2}+\frac{\rho^{2}}{4}\left\{\left(\partial_{a} \boldsymbol{p}\right)^{2}+\left(\partial_{a} \boldsymbol{q}\right)^{2}\right\} \\
& +\frac{1}{4} \rho^{2}\left\{n_{+}\left(\partial_{a} \hat{\bar{e}}_{b}\right)^{2}+n_{-}\left(\partial_{a} \hat{e}_{b}\right)^{2}\right\}+\frac{3}{8}\left(1-n_{3}^{2}\right) \rho^{4}-\frac{3}{8} \rho^{4}
\end{aligned}
$$

where

$$
\mathcal{F}_{a b}=\partial_{a} J_{b}-\partial_{b} J_{a}+\frac{1}{2} \boldsymbol{n} \cdot \partial_{a} \boldsymbol{n} \times \partial_{b} \boldsymbol{n}-\left\{\partial_{a}\left(n_{3} \hat{C}_{b}\right)-\partial_{b}\left(n_{3} \hat{C}_{a}\right)\right\}-2 \rho^{2} n_{3} H_{a b}
$$

We find it noteworthy that the final Lagrangian (60) contains only $U_{C}(1)$ and $U_{I}(1)$ invariant quantities, despite the fact that in (48) we have only introduced gauge fixing for the off-diagonal components $X_{a}^{ \pm}$. In particular, the $U_{C}(1) \in S U(2)$ gauge invariance has been eliminated explicitely by the introduction of gauge invariant variables. This elimination of the $U_{C}(1) \times U_{I}(1)$ gauge invariance has been at the expense of introducing variables $\hat{C}_{a}, \hat{\boldsymbol{e}}$ and $n_{ \pm}$which transform according to a projective representation of the $S O(4)$ (Lorentz) group. However, in (60) these variables appear only in $S O(4)$ invariant combinations.

The final Lagrangian (60) has a very interesting structure. It describes the interacting dynamics between a version of the $O(3)$ nonlinear $\sigma$-model that one of us introduced in 6] and the $G(4,2)$ Grassmannian nonlinear $\sigma$-model.

Clearly, the natural interpretation of the real scalar field $\rho$ is in terms of a condensate. Since $\rho$ is a positive definite quantity we can expect that it develops the non-vanishing ground state expectation value (24) that characterizes a material background in (60); see [19], 21].

Due to the presence of the third term in (60), a nonvanishing $\Delta$ in (24) leads to an effective mass to the vector field $J_{a}$. As a consequence this vector field is subject to the Meissner effect. If we assume that at large distances we can ignore the contribution from $J_{a}$, the remaining Lagrangian involves only variables that describe a the present version of the $O(3) \sigma$-model and the $G(4,2)$ Grassmannian non-linear $\sigma$-model. 
In the London limit where we replace $\rho$ by its ground state expectation value (24), the version of the $O(3)$ nonlinear $\sigma$-model that has been embedded in (60) has the following Lagrangian,

$$
\frac{\Delta^{2}}{8}\left(\mathrm{D}_{a}^{\hat{C}} \boldsymbol{n}\right)^{2}+\frac{1}{16}\left\{\boldsymbol{n} \cdot \partial_{a} \boldsymbol{n} \times \partial_{b} \boldsymbol{n}-2 \cdot\left\{\partial_{a}\left(n_{3} \hat{C}_{b}\right)-\partial_{b}\left(n_{3} \hat{C}_{a}\right)\right\}\right\}^{2}+\frac{3}{8} \Delta^{2}\left(1-n_{3}^{2}\right)
$$

This is in close resemblance with the effective Lagrangian (1), which we have proposed previously could be an effective model for $S U(2)$ Yang-Mills theory [3]- [5] . The difference stems from the fact that here the \pm components of the order parameter $\boldsymbol{n}$ lack Lorentz invariance due to the one-cocycle (32). The Lorentz invariance of the Lagrangian is restored by the presence of the similarly Lorentz invariance violating vector field $\hat{C}_{a}$.

We note that the last term in (61) is an additional $O(3)$ symmetry breaking potential term. It is Lorentz invariant since $n_{3}$ is the sole component of $\boldsymbol{n}$ that is a scalar under Lorentz transformations.

The reason for the particular combination of the potential terms that we have introduced in (46) (the third and fourth terms) becomes now obvious: This combination ensures that the angular variable $\theta$ of $\boldsymbol{n}$ in the parametrization (43) acquires a positive mass term. This choice still leaves the potential term involving only $\rho$ with a negative sign. Eventually, this sign will also find an explanation.

The original model (11) supports knotted closed strings as stable solitons. The version (61) involves also the dynamical gauge field $\hat{C}_{a}$ that restores Lorentz invariance in the present case. It would be very interesting to understand how the addition of this field affects the soliton structure of the theory.

We now proceed to identify the $G(4,2)$ nonlinear $\sigma$-model that has been embedded in (60). This embedding is determined by the kinetic term (54) that can be written as

$$
\left|\left(\partial_{a}+i C_{a}\right) \boldsymbol{e}\right|^{2}=\frac{\rho^{2}}{2}\left\{\left(\partial_{a} \boldsymbol{p}\right)^{2}+\left(\partial_{a} \boldsymbol{q}\right)^{2}\right\}
$$

This reveals the topological

$$
G(4,2) \sim \frac{S O(4)}{S O(2) \times S O(2)} \sim \mathbb{S}^{2} \times \mathbb{S}^{2}
$$


structure of the Grassmannian. We conclude that when we subject $\boldsymbol{p}$ and $\boldsymbol{q}$ to the two conditions (27), these two three-component vector fields describe the four dimensional Grassmannian manifold $G(4,2)$. Indeed, a priori the two vector fields $\boldsymbol{p}$ and $\boldsymbol{q}$ have six independent components. But due to the two conditions (27) only four of the components are independent, and correspond to coordinates on the four dimensional Grassmannian manifold $G(4,2)$.

Now, we return to the two terms (55) and (56) which together with the last term in (57) describe the coupling between the Grassmannian model and the $O(3)$ model. We argue that the Grassmannian contribution in the interaction terms (55) and (56) can be identified as the (anti)holomorphic one-form on the complex manifold $G(4,2) \sim \mathbb{S}^{2} \times \mathbb{S}^{2}$. For this we introduce the explicit parametrization (38), (39). When we specify to the magnetic limit where $\vartheta \rightarrow \pi / 2$ in (34) we find for the Grassmannian contribution in (55)

$$
\frac{1}{|\boldsymbol{s}|} \cdot<\partial_{a}(\boldsymbol{p}+\boldsymbol{q}), \boldsymbol{p}-\boldsymbol{q}-4 \sqrt{2} i \boldsymbol{s}>\stackrel{\vartheta \rightarrow 0}{\longrightarrow}-2 e^{i \psi}(\boldsymbol{l}+i \boldsymbol{m}) \cdot \partial_{a} \boldsymbol{k}=-2 e^{i \psi}(d \beta+i \sin \beta d \alpha)
$$

This is the (unique) holomorphic one-form on the magnetic two-sphere described by $\boldsymbol{k}$. Similarly we find in the electric limit $\vartheta \rightarrow 0$ that the Grassmannian contribution to (55), (56) yields the (anti)holomorphic one-form on the electric two-sphere in $G(4,2) \sim \mathbb{S}^{2} \times \mathbb{S}^{2}$ which is described by the unit vector $\boldsymbol{l}$. These observations endorse our proposal that the Grassmannian contributions in (55), (56) engage the holomorphic and the antiholomorphic one-forms on the complex manifold $G(4,2) \sim \mathbb{S}^{2} \times \mathbb{S}^{2}$.

Notice that in the interaction terms (55), (56) the phase $\psi$ in (62) can be combined with the phase of $n_{ \pm}$into

$$
\phi+2 \eta \rightarrow \phi+2 \eta+\psi
$$

Finally, for the last term in (157) we have in the Lorentz invariant ground state where $n_{3}= \pm 1$

$$
\frac{1}{4}\left(2 \rho^{2} n_{3} H_{a b}\right)^{2}=\frac{1}{2} n_{3}^{2} \rho^{4} \approx \frac{1}{2} \rho^{4}
$$


When we compare this with the last term in (60) we conclude that despite the negative sign of this term we have an overall stability of the theory. 


\section{GAUGE COVARIANCE}

Our description of the spin-charge separation employs the Pauli frame (15), (6) that identifies the diagonal matrix $\sigma^{3}$ with the direction of the $U_{C}(1)$ subalgebra in the $S U(2)$ Lie algebra. We now proceed to show that the spin-charge separation is frame

independent, instead of $\sigma^{3}$ we can select the direction of the Cartan subalgebra $U_{C}(1)$ in $S U(2)$ in an arbitrary and space-time dependent manner. For this we introduce an $a$ priori arbitrary $g(x) \in S U(2)$ and perform the conjugation

$$
\begin{gathered}
\sigma^{3} \stackrel{g}{\longrightarrow} g \sigma^{3} g^{-1} \stackrel{\text { def }}{=} m_{i} \sigma^{i}=\hat{\boldsymbol{m}} \\
\sigma^{ \pm} \stackrel{g}{\longrightarrow} g \sigma^{ \pm} g^{-1} \stackrel{\text { def }}{=} e_{i}^{ \pm} \sigma^{i} \stackrel{\text { def }}{=} \frac{1}{2}\left(e_{i}^{1} \pm i e_{i}^{2}\right) \sigma^{i}=\hat{\boldsymbol{e}}^{ \pm}
\end{gathered}
$$

Clearly, these matrices also satisfy the same algebra as (15), with $\hat{\boldsymbol{m}}$ the Cartan generator

$$
\begin{gathered}
{\left[\hat{\boldsymbol{m}}, \hat{\boldsymbol{e}}^{ \pm}\right]= \pm 2 \hat{\boldsymbol{e}}^{ \pm}} \\
{\left[\hat{\boldsymbol{e}}^{+}, \hat{\boldsymbol{e}}^{-}\right]=\hat{\boldsymbol{m}}}
\end{gathered}
$$

The gauge transformation (77) by the matrix $g$ maps (6) onto

$$
A_{a} \sigma^{3}+X_{a}^{+} \sigma^{+}+X_{a}^{-} \sigma^{-} \rightarrow A_{a} \hat{\boldsymbol{m}}+X_{a}^{+} \hat{\boldsymbol{e}}^{+}+X_{a}^{-} \hat{\boldsymbol{e}}^{-}+\mathfrak{a}_{a} \hat{\boldsymbol{m}}+\frac{1}{2 i}\left[\partial_{a} \hat{\boldsymbol{m}}, \hat{\boldsymbol{m}}\right]
$$

where

$$
\mathfrak{a}_{a}=-i \operatorname{tr}\left[\sigma^{3} g^{-1} \partial_{a} g\right]
$$

We interpret this connection in the following manner: We define

$$
\begin{gathered}
\mathcal{A}_{a}=\mathcal{A}_{a}^{i} \sigma^{i}=\left(A_{a}+\mathfrak{a}_{a}\right) \hat{\boldsymbol{m}}+\frac{1}{2 i}\left[\partial_{a} \hat{\boldsymbol{m}}, \hat{\boldsymbol{m}}\right]=g A_{a} \sigma^{3} g^{-1}+2 i g \partial_{a} g^{-1} \\
=C_{a} \hat{\boldsymbol{m}}+\frac{1}{2 i}\left[\partial_{a} \hat{\boldsymbol{m}}, \hat{\boldsymbol{m}}\right]
\end{gathered}
$$

and

$$
\mathcal{X}_{a}=\mathcal{X}_{a}^{i} \sigma^{i}=X_{a}^{+} \hat{\boldsymbol{e}}^{+}+X_{a}^{-} \hat{\boldsymbol{e}}^{-}=g\left(X_{a}^{+} \sigma^{+}+X_{a}^{-} \sigma^{-}\right) g^{-1}
$$

Here $\mathcal{A}_{a}$ is the connection originally introduced by Duan and Ge [10], and subsequently by Cho [11]; see also [3]. 
We introduce a generic $h(x) \in S U(2)$ and redefine

$$
g \rightarrow g h
$$

This determines a transformation under which $\mathcal{A}$ transforms as a connection

$$
\mathcal{A} \rightarrow h \mathcal{A} h^{-1}+2 i h d h^{-1}
$$

while $\mathcal{X}$ transforms as a tensor,

$$
\mathcal{X} \rightarrow h \mathcal{X} h^{-1}
$$

In (63), (64) we have superficially fourteen field degrees of freedom. These are the four components of $C_{a}$, the eight components of $X_{a}^{ \pm}$and the two independent components of $\hat{\boldsymbol{m}}$. However, if we impose the $h$-covariant condition [24]

$$
\mathcal{D}[\mathcal{A}]_{a}^{i j} \mathcal{X}_{a}^{j}=0
$$

this condition eliminates two of the field variables and we are left with only the twelve independent components of a four dimensional $S U(2)$ gauge field.

The condition (65) is a gauge covariant version of the maximal abelian gauge condition (49). Explicitely, when we substitute (63) and (64) in (65) and use the identity

$$
m^{i}\left(\delta^{i j} \partial_{a}+\epsilon^{i k j} \mathcal{A}_{a}^{k}\right) \mathcal{X}_{a}^{j}=0
$$

we conclude that (65) is equivalent to the condition (49) for the original components $\left(A_{\mu}, X_{\mu}^{ \pm}\right)$. In particular, when we choose $\hat{\boldsymbol{m}} \equiv \sigma^{3}$ we find that (65) reduces to (49) and we retain all our previous results. This confirms that our separation between the spin and the charge in the Yang-Mills Lagrangian is gauge covariant, independent of the direction of $U_{C}(1)$ in the $S U(2)$ gauge group. Furthermore, the connection by Duan and Ge and by Cho acquires a role in the gauge covariantization of our formalism. 


\section{CONFORMAL GEOMETRY}

The Yang-Mills Lagrangian has a number of attractive features that become transparent when we present it in terms of the independent spin and charge variables. For example, the Lagrangian can be related to a two-gap superconductor model 20], and $\rho$ admits also an independent interpretation as a gauge invariant condensate [19], 21].

Here we shall propose an alternative interpretation of the Yang-Mills Lagrangian. We shall propose that $\rho$ can be viewed as the conformal scale of a conformally flat metric tensor, and (60) describes the coupling between matter fields and the Einstein-Hilbert gravity in the presence of a nontrivial cosmological constant.

The version of the spin-charge separated Yang-Mills Lagrangian that we shall employ is the following,

$$
L_{Y M}=L_{Y M}^{(1)}+L_{Y M}^{(2)}+L_{Y M}^{(3)}+L_{Y M}^{(4)}
$$

where

$$
\begin{aligned}
& L_{Y M}^{(1)}=\frac{1}{4}\left\{\partial_{a} J_{b}-\partial_{b} J_{a}+\frac{1}{2} \boldsymbol{n} \cdot \mathrm{D}_{a}^{\hat{C}} \boldsymbol{n} \times \mathrm{D}_{b}^{\hat{C}} \boldsymbol{n}-n_{3}\left(\partial_{a} \hat{C}_{b}-\partial_{b} \hat{C}_{a}\right)-2 \rho^{2} n_{3} H_{a b}\right\}^{2} \\
& L_{Y M}^{(2)}=\frac{1}{2} \rho^{2} J_{a}^{2}+\frac{1}{8} \rho^{2}\left(\mathrm{D}_{a}^{\hat{C}} \boldsymbol{n}\right)^{2} \\
& L_{Y M}^{(3)}=\rho^{2}\left|\mathrm{D}_{A a}^{C} e_{b}\right|^{2}+\frac{1}{2} \rho^{2} t_{+}\left(\overline{\mathrm{D}}_{A a}^{C} \bar{e}_{b}\right)^{2}+\frac{1}{2} \rho^{2} t_{-}\left(\mathrm{D}_{A a}^{C} e_{b}\right)^{2} \\
& L_{Y M}^{(4)}=\frac{1}{2}\left(\partial_{a} \rho\right)^{2}+\frac{3}{8}\left(1-n_{3}^{2}\right) \rho^{4}-\frac{3}{8} \rho^{4}
\end{aligned}
$$

Our goal is to write these terms in a manifestly covariant manner, with the conformally flat metric tensor

$$
g_{\mu \nu}=\left(\frac{\rho}{\Delta}\right)^{2} \delta_{\mu \nu}
$$

Here $\Delta$ is a constant with dimensions of mass: Since $\rho$ has dimensions of mass we need to introduce $\Delta$ so that the components of the metric tensor acquire their correct 
dimensionality. The obvious choice is to identify $\Delta$ with the vacuum expectation value of the condensate $\rho$ according to (24).

We introduce the vierbein

$$
g_{\mu \nu}=\delta_{a b} E_{\mu}^{a} E^{b}
$$

where

$$
\delta^{a b}=g^{\mu \nu} E_{\mu}^{a} E_{\nu}^{b}
$$

and the vierbein $E^{a}{ }_{\mu}$ is given explicitely by

$$
E_{\mu}^{a}=\frac{\rho}{\Delta} \delta_{\mu}^{a}
$$

with

$$
E^{a}{ }_{\mu} E_{b}{ }^{\mu}=\delta^{a}{ }_{b}
$$

The Christoffel symbol of the metric (170) is

$$
\Gamma_{\nu \sigma}^{\mu}=\frac{1}{2} g^{\mu \eta}\left(\partial_{\nu} g_{\eta \sigma}+\partial_{\sigma} g_{\eta \nu}-\partial_{\eta} g_{\nu \sigma}\right)=\frac{1}{4}\left\{\delta_{\sigma}^{\mu} \delta_{\nu}^{\tau}+\delta_{\nu}^{\mu} \delta_{\sigma}^{\tau}-\delta^{\mu \tau} \delta_{\nu \sigma}\right\} \partial_{\tau} \ln \sqrt{g}
$$

where

$$
\sqrt{g}=\left(\frac{\rho}{\Delta}\right)^{4}
$$

The spin connection is defined by demanding covariant constancy of the vierbein,

$$
\partial_{\mu} E_{a}{ }^{\nu}+\Gamma_{\mu \lambda}^{\nu} E_{a}^{\lambda}-\omega_{\mu a}^{b} E_{b}^{\nu}=0
$$

This gives

$$
\begin{aligned}
\omega_{\mu b}^{a} & =E^{a}{ }_{\nu} \nabla_{\mu} E_{b}{ }^{\nu}=E^{a}{ }_{\nu}\left(\partial_{\mu} E_{b}{ }^{\nu}+\Gamma_{\mu \lambda}^{\nu} E_{b}{ }^{\lambda}\right) \\
& =-E_{b}{ }^{\nu} \nabla_{\mu} E^{a}{ }_{\nu}=-E_{b}{ }^{\nu}\left(\partial_{\mu} E^{a}{ }_{\nu}-\Gamma_{\mu \nu}^{\lambda} E^{a}{ }_{\lambda}\right)
\end{aligned}
$$

In these relations we also indicate how the covariant derivative $\nabla_{\mu}$ acts on the vector and co-vector fields.

Explicitely we get from the metric tensor (70), (71) for the spin connection

$$
\omega_{\mu b}^{a}=\frac{1}{4}\left\{\delta_{\mu}^{a} \delta_{b}{ }^{\sigma}-\delta_{b d} \delta^{d}{ }_{\mu} \delta^{a c} \delta_{c}{ }^{\sigma}\right\} \partial_{\sigma} \ln \sqrt{g}
$$


We employ the vierbein $E^{a}{ }_{\mu}$ and the complex Grassmannian zweibein (15) to introduce the following complex zweibein

$$
\begin{aligned}
\mathbb{e}_{\mu} & =E^{a}{ }_{\mu} e_{a} \\
\overline{\mathbb{e}}_{\mu} & =E^{a}{ }_{\mu} e_{a}^{*}
\end{aligned}
$$

This zweibein is then normalized w.r.t. the metric $g_{\mu \nu}$ according to

$$
\begin{gathered}
g^{\mu \nu} \mathbb{e}_{\mu} \overline{\mathbb{E}}_{\nu}^{*}=1 \\
g^{\mu \nu} \mathbb{e}_{\mu} \mathbb{e}_{\nu}=g^{\mu \nu} \overline{\mathbb{E}}_{\mu}^{*} \overline{\mathbb{E}}_{\nu}^{*}=0
\end{gathered}
$$

When we push forward the spin connection into

$$
\omega_{\mu b}^{a} \rightarrow \omega_{\mu \nu}^{\lambda}=E_{a}^{\lambda} \omega_{\mu b}^{a} E_{\nu}^{b}
$$

we can introduce the generally covariant version of the connection (23),

$$
\mathcal{C}_{\mu}=i \overline{\mathbb{e}}^{\sigma}\left(\partial_{\mu} \mathbb{e}_{\sigma}-\Gamma_{\mu \sigma}^{\lambda} \mathbb{e}_{\lambda}+\omega_{\mu \sigma}^{\lambda} \mathbb{e}_{\lambda}\right)=i \overline{\mathbb{e}}^{\sigma} \nabla_{\mu} \mathbb{e}_{\sigma}+i \overline{\mathbb{e}}^{\lambda} \omega_{\mu \lambda}^{\sigma} \mathbb{e}_{\sigma}
$$

and when we twist the covariant derivative operator with (74),

$$
\nabla_{\mu}^{\mathcal{C}}=\nabla_{\mu}+i \mathcal{C}_{\mu}
$$

we have an operator that parallel transports the zweibein.

We now proceed to employ this formalism to rewrite the Yang-Mills Lagrangian (66)(69) in a generally covariant manner. Our computations simplify considerably when we observe that for the metric tensor (70)

$$
\nabla_{\mu} \mathbb{e}_{\nu}+\omega_{\mu \nu}^{\lambda} \mathbb{e}_{\lambda}=\partial_{\mu} \mathbb{e}_{\nu}-\Gamma_{\mu \nu}^{\lambda} \mathbb{e}_{\lambda}+\omega_{\mu \nu}^{\lambda} \mathbb{e}_{\lambda}=\rho \cdot \partial_{\mu}\left(\frac{\mathbb{e}_{\nu}}{\rho}\right)
$$

and

$$
\mathcal{C}_{\mu}=i \overline{\mathrm{e}}^{\nu} \partial_{\mu} \mathbb{e}_{\nu}-\frac{i}{4} \partial_{\mu} \ln \sqrt{g}
$$

We start with the second and third term in (66), which we write in a generally covariant form as follows,

$$
\frac{1}{2} \boldsymbol{n} \cdot \mathrm{D}_{a}^{\hat{C}} \boldsymbol{n} \times \mathrm{D}_{b}^{\hat{C}} \boldsymbol{n}-n_{3}\left(\partial_{a} \hat{C}_{b}-\partial_{b} \hat{C}_{a}\right) \rightarrow \frac{1}{2} \boldsymbol{n} \cdot \nabla_{\mu}^{\mathcal{C}} \boldsymbol{n} \times \nabla_{\nu}^{\mathcal{C}} \boldsymbol{n}-n_{3}\left(\partial_{\mu} \mathcal{C}_{\nu}-\partial_{\nu} \mathcal{C}_{\mu}\right)
$$


We also write the last contribution in (66) as

$$
-2 \rho^{2} n_{3} H_{a b}=-i \rho^{2} n_{3}\left(e_{a} e_{b}^{*}-e_{b} e_{a}^{*}\right) \rightarrow-i \Delta \cdot n_{3}\left(\mathbb{e}_{\mu} \mathbb{e}_{\nu}^{*}-\mathbb{e}_{\nu} \mathbb{e}_{\mu}^{*}\right)=-2 \Delta \cdot n_{3} \mathcal{H}_{\mu \nu}
$$

When we define

$$
\mathcal{F}_{\mu \nu}=\partial_{\mu} J_{\nu}-\partial_{\nu} J_{\mu}+\frac{1}{2} \boldsymbol{n} \cdot\left\{\nabla_{\mu}^{\mathcal{C}} \boldsymbol{n} \times \nabla_{\nu}^{\mathcal{C}} \boldsymbol{n}-2 \hat{\boldsymbol{z}}\left[\left(\partial_{\mu} \mathcal{C}_{\nu}-\partial_{\nu} \mathcal{C}_{\mu}\right)+2 \Delta \cdot \mathcal{H}_{\mu \nu}\right]\right\}
$$

where $\hat{\boldsymbol{z}}$ is a unit vector in the $z$-direction of the internal space, we conclude that we can write the entire (66) in the following generally covariant form

$$
L_{Y M}^{(1)} \rightarrow \mathcal{L}_{Y M}^{(1)}=\frac{1}{4} \sqrt{g} g^{\mu \nu} g^{\rho \sigma} \mathcal{F}_{\mu \rho} \mathcal{F}_{\nu \sigma}
$$

Similarly we can write (67) in the following generally covariant form,

$$
L_{Y M}^{(2)} \rightarrow \mathcal{L}_{Y M}^{(2)}=\Delta^{2} \cdot \sqrt{g} g^{\mu \nu}\left(J_{\mu} J_{\nu}+\nabla_{\mu}^{\mathcal{C}} \boldsymbol{n} \cdot \nabla_{\nu}^{\mathcal{C}} \boldsymbol{n}\right)
$$

We now proceed to (68). For this we send our flat space $U_{I}(1)$ covariant derivative of the Euclidean metric Grassmannian zweibein to a generally covariant form as follows,

$$
\left(\partial_{a}+i C_{a}\right) e_{b} \rightarrow E_{a}^{\lambda}\left\{\delta_{\lambda}^{\nu} \nabla_{\mu}^{\mathcal{C}}+\omega_{\mu \lambda}^{\nu}\right\} \mathbb{e}_{\nu}=E_{a}{ }^{\lambda} \mathcal{D}_{\mu \lambda}^{\nu} \mathbb{e}_{\nu}
$$

We have here introduced the following twisted covariant derivative

$$
\mathcal{D}_{\mu \lambda}^{\nu}=\delta_{\lambda}^{\nu} \nabla_{\mu}^{\mathcal{C}}+\omega_{\mu \lambda}^{\nu}
$$

It extends the action of the twisted covariant derivative (75) to the vector fields $\mathbb{e}_{\nu}$. With this, we can present the entire (68) in the following covariant form,

$$
\begin{gathered}
L_{Y M}^{(3)} \rightarrow \mathcal{L}_{Y M}^{(3)} \\
=\Delta^{2} \cdot \sqrt{g} \cdot g^{\mu \nu} g^{\lambda \eta}\left\{\left(\overline{\mathcal{D}}_{\mu \lambda}^{\sigma} \overline{\mathbb{E}}_{\sigma}\right)\left(\mathcal{D}_{\nu \eta}^{\kappa} \mathbb{e}_{\kappa}\right)+\frac{1}{2} t_{+}\left(\overline{\mathcal{D}}_{\mu \lambda}^{\sigma} \overline{\mathbb{E}}_{\sigma}\right)\left(\overline{\mathcal{D}}_{\nu \eta}^{\kappa} \overline{\mathbb{e}}_{\kappa}\right)+\frac{1}{2} t_{-}\left(\mathcal{D}_{\mu \lambda}^{\sigma} \mathbb{e}_{\sigma}\right)\left(\mathcal{D}_{\nu \eta}^{\kappa} \mathbb{e}_{\kappa}\right)\right\}
\end{gathered}
$$


Finally, we proceed to (69). We introduce the Riemann tensor in terms of the Christofffel symbol (172)

$$
R_{\mu \nu \sigma}^{\lambda}=\partial_{\nu} \Gamma_{\mu \sigma}^{\lambda}-\partial_{\mu} \Gamma_{\nu \sigma}^{\lambda}+\Gamma_{\mu \sigma}^{\eta} \Gamma_{\eta \nu}^{\lambda}-\Gamma_{\nu \sigma}^{\eta} \Gamma_{\eta \mu}^{\lambda}
$$

and the Ricci tensor

$$
R_{\mu \nu}=R_{\nu \mu}=R_{\mu \lambda \nu}^{\lambda}
$$

and the Ricci scalar

$$
R=R_{\mu}^{\mu}
$$

which transforms according to (in $D$ dimensional space)

$$
R \rightarrow \tilde{R}=\phi^{-2}\left\{R-2(D-1) g^{\mu \nu} \nabla_{\mu} \nabla_{\nu} \ln \phi-(D-2)(D-1) g^{\mu \nu}\left(\nabla_{\mu} \ln \phi\right)\left(\nabla_{\nu} \ln \phi\right)\right\}
$$

under the conformal scaling

$$
g_{\mu \nu} \rightarrow \tilde{g}_{\mu \nu}=\phi^{2} g_{\mu \nu}
$$

of the metric tensor. For the metric tensor (70) this leads to the identification

$$
\frac{1}{2}\left(\partial_{\mu} \rho\right)^{2} \rightarrow \frac{1}{\Delta^{2}} \sqrt{g} R
$$

This is the covariant interpretation of the first term in (69). For the remaining terms in (69) (except for the surface term) we get from (170)

$$
\frac{3}{8}\left(1-n_{3}^{2}\right) \rho^{4}-\frac{3}{8} \rho^{4} \rightarrow \frac{1}{\Delta^{2}} \frac{3}{8}\left(1-n_{3}^{2}\right) \sqrt{g}-\frac{1}{\Delta^{2}} \frac{3}{8} \sqrt{g}
$$

and we conclude that the entire (69) can be presented in the following generally covariant manner,

$$
L_{Y M}^{(4)} \rightarrow \mathcal{L}_{Y M}^{(4)}=\frac{1}{\Delta^{2}} \sqrt{g} R-\frac{3}{8} \frac{1}{\Delta^{2}} \sqrt{g}+\frac{3}{8} \frac{1}{\Delta^{2}}\left(1-n_{3}^{2}\right) \sqrt{g}
$$

Here the first contribution is the standard Einstein-Hilbert Lagrangian, the second is the standard (negative) cosmological constant term, and the third gives a (in general) space-time dependent correction to the cosmological constant when $n_{3} \neq \pm 1$. 
Note that the sign of the Ricci scalar is consistent with the sign proposed in [25], ensuring that the Euclidean Einstein-Hilbert Lagrangian is bounded from below.

We conclude by summarizing, that when we combine (176), (177), (178) and (180) we find that in terms of the spin-charge separated variables the Yang-Mills Lagrangian can be written in the following generally covariant form

$$
L_{Y M}=\mathcal{L}_{Y M}^{(1)}+\mathcal{L}_{Y M}^{(2)}+\mathcal{L}_{Y M}^{(3)}+\mathcal{L}_{Y M}^{(4)}
$$

Here

$$
\mathcal{L}_{Y M}^{(1)}=\frac{1}{4} \sqrt{g} g^{\mu \nu} g^{\rho \sigma} \mathcal{F}_{\mu \rho} \mathcal{F}_{\nu \sigma}
$$

which has the standard form of the generally covariant Maxwell Lagrangian,

$$
\mathcal{L}_{Y M}^{(2)}=\Delta^{2} \cdot \sqrt{g} g^{\mu \nu}\left(J_{\mu} J_{\nu}+\nabla_{\mu}^{\mathcal{C}} \boldsymbol{n} \cdot \nabla_{\nu}^{\mathcal{C}} \boldsymbol{n}\right)
$$

is a generally covariant current-current interaction term,

$\mathcal{L}_{Y M}^{(3)}=\Delta^{2} \cdot \sqrt{g} \cdot g^{\mu \nu} g^{\lambda \eta}\left\{\left(\overline{\mathcal{D}}_{\mu \lambda}^{\sigma} \overline{\mathbb{E}}_{\sigma}\right)\left(\mathcal{D}_{\nu \eta}^{\kappa} \mathbb{e}_{\kappa}\right)+\frac{1}{2} t_{+}\left(\overline{\mathcal{D}}_{\mu \lambda}^{\sigma} \overline{\mathbb{E}}_{\sigma}\right)\left(\overline{\mathcal{D}}_{\nu \eta}^{\kappa} \overline{\mathbb{E}}_{\kappa}\right)+\frac{1}{2} t_{-}\left(\mathcal{D}_{\mu \lambda}^{\sigma} \mathbb{e}_{\sigma}\right)\left(\mathcal{D}_{\nu \eta}^{\kappa} \mathbb{e}_{\kappa}\right)\right\}$

gives the kinetic term for the Grassmannian $\mathbb{e}_{\mu}$ together with two terms describing its interaction with $\boldsymbol{n}$ where we recall that these interaction terms can be related to the (anti)holomorphic one-forms on the Grassmannian. Finally,

$$
\mathcal{L}_{Y M}^{(4)}=\frac{1}{\Delta^{2}} \sqrt{g} R+-\frac{3}{8} \frac{1}{\Delta^{2}} \sqrt{g}+\frac{3}{8} \frac{1}{\Delta^{2}}\left(1-n_{3}^{2}\right) \sqrt{g}
$$

is the Einstein-Hilbert Lagrangian together with a cosmological constant with a spacetime dependent correction that vanishes in the ground state where $n_{3}= \pm 1$.

The final Lagrangian has a manifestly generally covariant form, and it coincides with the spin-charge separated Yang-Mills Lagrangian (66) -(69) when we evaluate it using the conformally flat metric tensor (170). Notice that from the present point of view the nontriviality of the ground state expectation value in (24) becomes quite natural. When $\rho$ vanishes our conformal space-time just shrinks away. 


\section{STATIC LIMIT}

It is often instructive to inspect the static limit of the Lagrangian, it gives an indication on the ground state properties of the theory. We reach the static limit when we only retain the spatial derivatives and set the time component of the vector field $\boldsymbol{e}$ to zero. This sends $\vartheta \rightarrow \pi / 2$ in (34), and as a consequence the electric vector field $\boldsymbol{p}$ vanishes and the only non-vanishing contribution to the tensor field $H_{a b}$ is

$$
H_{i j}=\frac{1}{2 \sqrt{2}} \epsilon_{i j k} l_{k}
$$

where $\boldsymbol{l}$ is the unit vector in the magnetic direction. Furthermore, from (36), (37), (42) we get

$$
\partial_{i} \hat{C}_{j}-\partial_{j} \hat{C}_{i}=-\frac{1}{2 \sqrt{2}} \boldsymbol{l} \cdot \partial_{i} \boldsymbol{l} \times \partial_{j} \boldsymbol{l}
$$

and

$$
\left(\partial_{i} e_{j}\right)^{2}=\frac{1}{16} \cdot\left\{(\boldsymbol{l}+i \boldsymbol{m}) \cdot \partial_{i} \boldsymbol{k}\right\}^{2}
$$

With these, we get from (60) for the energy density in the static limit

$$
\begin{gathered}
H_{\text {static }}=\frac{1}{2}\left(\partial_{i} \rho\right)^{2}+\frac{1}{2} \rho^{2} J_{i}^{2}+\frac{1}{8} \rho^{2}\left(\mathrm{D}_{i}^{\hat{C}} \boldsymbol{n}\right)^{2}+\frac{1}{32} \rho^{2}\left(\partial_{i} \boldsymbol{l}\right)^{2} \\
+\frac{1}{64} \rho^{2}\left\{n_{+} e^{-2 i \psi}\left([\boldsymbol{l}+i \boldsymbol{m}] \cdot \partial_{i} \boldsymbol{k}\right)^{2}+n_{-} e^{2 i \psi}\left([\boldsymbol{l}-i \boldsymbol{m}] \cdot \partial_{i} \boldsymbol{k}\right)^{2}\right\} \\
+\frac{1}{4} \mathcal{F}_{i j}^{2}+\frac{3}{8}\left(1-n_{3}^{2}\right) \rho^{4}-\frac{3}{8} \rho^{4}
\end{gathered}
$$

Here

$$
\mathcal{F}_{i j}=\partial_{i} J_{j}-\partial_{j} J_{i}+\frac{1}{2} \boldsymbol{n} \cdot \mathrm{D}_{i}^{\hat{C}} \boldsymbol{n} \times \mathrm{D}_{j}^{\hat{C}} \boldsymbol{n}+n_{3} \frac{1}{2 \sqrt{2}}\left\{\boldsymbol{l} \cdot \partial_{i} \boldsymbol{l} \times \partial_{j} \boldsymbol{l}-2 \rho^{2} \epsilon_{i j k} l_{k}\right\}
$$

From this we can draw the following conclusions:

There is an apparent duality between the internal vector field $\boldsymbol{n}$ and the space-valued vector field $\boldsymbol{l}$. In particular, both are embedded in (81)-(83) in a manner that employs the version (11) of the $O(3)$ nonlinear $\sigma$-model. As a consequence both $\boldsymbol{n}$ and $\boldsymbol{l}$ have the potential of supporting closed knotted strings as stable solitons. It is suggestive to 
interpret $\boldsymbol{l}$ as a "magnetic" order parameter, and $\boldsymbol{n}$ as an "electric" order parameter in the static limit [4].

The vector field $\boldsymbol{n}$ takes values in the internal space. Due to the one-cocycle (32) in the Lorentz transformations, it has a unique rotation invariant ground state value at large distances which is given by (44). But $\boldsymbol{l}$ is a space valued vector field, it transforms as a vector under spatial $S O(3)$ rotations. Consequently its only conceivable asymptotic ground state value at large distances is the spherically symmetric

$$
\boldsymbol{l} \stackrel{r \rightarrow \infty}{\longrightarrow} \frac{x}{r}
$$

From this we get the asymptotic behaviour

$$
\boldsymbol{l} \cdot \partial_{i} \boldsymbol{l} \times \partial_{j} \boldsymbol{l} \stackrel{r \rightarrow \infty}{\longrightarrow} \epsilon_{i j k} \frac{x^{k}}{r^{3}} \sim \frac{1}{r^{2}} \epsilon_{i j k} l_{k}
$$

for the third contribution to (84). Note that this is reminiscent of a magnetic monopole.

We combine the last two terms in (84) asymptotically into

$$
\boldsymbol{l} \cdot \partial_{i} \boldsymbol{l} \times \partial_{j} \boldsymbol{l}-2 \rho^{2} \epsilon_{i j k} l_{k} \stackrel{r \rightarrow \infty}{\longrightarrow}\left(\frac{1}{r^{2}}-2 \rho^{2}\right) \epsilon_{i j k} \frac{x^{k}}{r}
$$

We now make the following proposals: For a finite energy, we can expect that each of the positive definite terms in the static Hamiltonian are integrable. This means that asymptotically at large distances, in an analytic power expansion in $r$ we can expect

$$
\rho(r) \lesssim \mathcal{O}\left(\frac{1}{r}\right)
$$

In terms of the four dimensional metric tensor (70) this suggests that for finite energy the space should be compact. But from the present static point of view we can also argue as follows. We consider the space-time to have the topology of $\mathcal{M} \times \mathbb{R}^{1}$ where $\mathcal{M}$ is the three dimensional space manifold and $\mathbb{R}^{1}$ is the time. If we define the three-dimensional, spatial metric tensor by setting

$$
g_{i j}^{(3)}=\frac{\rho^{4}}{\Delta^{4}} \delta_{i j}
$$


we find that some of the terms in the energy density admit an independent, three dimensional geometric interpretation: From (729), the first term in the r.h.s. of (81) can be written in terms of the three dimensional Ricci scalar as

$$
\frac{1}{2}\left(\partial_{i} \rho\right)^{2}=\frac{1}{80} \frac{1}{\Delta^{2}} \sqrt{g^{(3)}} R^{(3)}
$$

The second term can be written as

$$
\frac{1}{2} \rho^{2} J_{i}^{2}=\frac{1}{2} \sqrt{g^{(3)}} g^{(3) i k} J_{i} J_{k}
$$

Similarly we conclude that each of the terms in (81) and (82) admit a generally covariant interpretation in terms of the present three dimensional conformal geometry.

For the terms in (83) the present three dimensional geometric interpretation appears to fail. But when we demand that the quadratic terms are independently integrable, since the asymptotic behaviour of $\boldsymbol{n}$ is dictated by (44) we can argue that it becomes very natural to expect that asymptotically the two terms in (85) cancel each other. This suggests that at large distances we have

$$
\rho^{2} \sim \frac{1}{2} \frac{1}{r^{2}+\lambda^{2}}
$$

where $\lambda$ is some parameter. When we substitute this in (86) we find that the spatial part of our space-time becomes asymptotically compactified into the sphere $\mathbb{S}^{3}$. It would be very interesting if this proposal could be made more rigorous. 


\section{SUMMARY}

We conclude our article with a summary of our results and a number of remarks on their possible physical consquences.

We have introduced a novel, complete field decomposition in the Yang-Mills Lagrangian. The decomposition implements a separation between the spin and the charge in the gauge field. The decomposition also introduces an internal, compact $U(1)$ interaction. A compact $U(1)$ gauge theory is known to exhibit confinement in a strong coupling domain which is separated from a weakly coupled and deconfined domain by a first order phase transition. Since the coupling in an abelian theory should increase when the distance scale decreases, the spin-charge separation is not in an apparent conflict with the high energy limit of the Yang-Mills theory, represented by asymptotically free and massless gauge bosons.

The spin-charge separated Yang-Mills Lagrangian describes the interacting dynamics between a version [6] of the $O(3)$ nonlinear $\sigma$-model and a $G(4,2)$ Grassmannian nonlinear $\sigma$-model, in a conformally flat spacetime and in the presence of both the Einstein-Hilbert Lagrangian and a negative cosmological constant term.

The conformal scale of the metric coincides with the gauge invariant condensate that has been studied previously in [19], 21]. Numerical lattice studies indicate that the ground state value (24) of the condensate is nonvanishing. From our geometrical point of view this is an expected result: If the conformal scale vanishes there is no space-time.

The metric properties of the classical Yang-Mills theory are consistent with the short distance limit of a renormalizable higher derivative gravitation theory with a Lagrangian of the form (3). It would be truly exciting if at short distance Einstein gravity metamorphoses into a Yang-Mills theory, as a single renormalizable quantum theory of material interactions.

The presence of the higher derivative term in (3) gives rise to a linearly increasing component in the large distance gravitational interaction. From the point of view of the Yang-Mills theory this may have some obvious advantages. However, at distance 
scales which are well beyond those that should be described by the Yang-Mills theory as such, it may become desirable for the $\beta$-function of the coupling $\gamma$ in (3) to force this coupling to flow towards $\gamma=0$. Such a large distance behaviour in the quantum theory would then leave the conventional Einstein gravity as the sole surviving very long range component of the spin-charge separated Yang-Mills theory.

Note that since $\gamma$ flows towards $\gamma \rightarrow \infty$ at short distances, the asymptotic condition (4) dissolves the massless modes of the Einstein gravity from the (not too) short distance spectrum. This leaves us with a gapped and spin-charge separated Yang-Mills theory that describes asymptotically free gauge vectors as distance scale goes to zero.

Our results suggest that due to fluctuations in the gauge invariant condensate $\rho$ and the vector field $\boldsymbol{n}$, at short distances both Newton's constant and the cosmological constant become variable.

The version of the $O(3)$ nonlinear $\sigma$ model that embeds the vector field $\boldsymbol{n}$ in the YangMills Lagrangian, essentially coincides with (11). This Lagrangian is known to describe knotted strings as stable solitons. Our results then support the proposal [3]-[5] that such strings are present in the spectrum of the $S U(2)$ Yang-Mills theory.

In the absence of the potential term in (11), the spectrum of the $O(3)$ model contains two massless Goldstone bosons. These bosons originate from the asymptotic breaking of the global $O(3)$ symmetry, when we select the large distance direction for the unit vector $\boldsymbol{s}$. In the case of the Yang-Mills theory, these massless Goldstone bosons are removed by the one-cocycle that breaks the Lorentz invariance of the pertinent order parameter $\boldsymbol{n}$. The requirement that the large distance ground state is Lorentz invariant uniquely fixes the asymptotic direction of the order parameter.

The spin-charge separated Yang-Mills theory also describes the $G(4,2)$ Grassmannian nonlinear $\sigma$-model. The $\mathbb{S}^{2} \times \mathbb{S}^{2}$ structure of the $G(4,2)$ manifold has an interpretation in terms of electric and magnetic variables, the two spheres $\mathbb{S}^{2}$ are related to each other by an electric-magnetic duality. In terms of the corresponding unit vector fields, the Grassmannian contribution to the Yang-Mills Lagrangian admits a very transparent 
realization. In particular, in the static (magnetic) limit we are left with only one threecomponent unit vector field $\boldsymbol{l}$, pointing in the magnetic direction. This vector field is embedded in the Yang-Mills Lagrangian by a version of the Lagrangian (11). Consequently we have an additional duality between two different embeddings of (1D), described by $\boldsymbol{n}$ and $\boldsymbol{l}$ respectively. In particular, this means that the vector field $\boldsymbol{l}$ has also the potential of supporting closed knotted strings as stable solitons.

The interaction between the $O(3) \sigma$-model and the Grassmannian $\sigma$ model involves the (anti)holomorphic one-forms on the Grassmannian manifold. These appear in a combination with the $n_{ \pm}$components of the vector field $\boldsymbol{n}$. The presence of the one-cocycle in the Lorentz transformation of $\boldsymbol{n}$ implies that at large distances these interaction terms are absent.

Finally, we have argued that demanding finiteness of energy in the Yang-Mills theory enforces a compactification of the space. We have proposed that it is natural for the asymptotic topology of the space-time to coincide with that of the manifold $\mathbb{S}^{3} \times \mathbb{R}^{1}$. 


\section{ACKNOWLEDGEMENTS}

The work by L.D.F. has been supported by RFBR grant 05-01-00922, CRDF grant RUM-1-2622-ST-04, and the program "Problems of nonlinear dynamics" of Presidium of Russian Academy of Sciences. The work by A.J.N. has been supported by a Grant from ANR, by a VR Grant, by a STINT Institutional Grant and by a STINT Thunberg Stipend. L.D.F. thanks L. Lipatov and A. Slavnov for discussions. A.J.N. thanks M. Chernodub, U. Danielsson, U. Lindström, M. Volkov and K. Zarembo for discussions, M. Niedermaier, M. Paranjape and A. Tseytlin for communications, and S. Slizovskiy for comments. A.J.N. also thanks the Yukawa Institute at the Kyoto University, the Department of Physics at Tokyo University and the Asian Pasific Center for Theoretical Physics for hospitality during the early part of this work. 
[1] A private communication by C.N. Yang.

[2] see the website http://www.claymath.org/millennium/ for a general discussion.

[3] L.D. Faddeev and A.J. Niemi, Phys. Rev. Lett. 82 (1999) 1624; L.D. Faddeev and A.J. Niemi, Phys. Lett. B449 (1999);

[4] L.D. Faddeev and A.J. Niemi, Phys. Lett. B525 (2002) 195;

[5] L.D. Faddeev, A.J. Niemi and U. Wiedner, Phys. Rev. D70 (2004) 114033

[6] L. Faddeev, Quantisation of Solitons, preprint IAS Print-75-QS70, 1975; and in Einstein and Several Contemporary Tendencies in the Field Theory of Elementary Particles in Relativity, Quanta and Cosmology vol. 1, M. Pantaleo, F. De Finis (eds.), Johnson Reprint, 1979

[7] R. Battye and P. Sutcliffe, Proc. Roy. Soc. London A455 (1999) 4305; Phys. Rev. Lett. 81 (1998) 4798; J. Hietarinta and P. Salo, Phys. Lett. B451 (1999) 60

[8] F. Lin and Y. Yang, Comm. Math. Phys. 249 (2004) 273

[9] L.D. Faddeev and A.J. Niemi, Nature 387 (1997) 58

[10] Y.S. Duan and M.L. Ge, Sinica Sci. 11 (1979) 1072

[11] Y.M. Cho, Phys. Rev. D21 (1980) 1080; Y.M. Cho, Phys. Rev. Lett. 46 (1981) 302

[12] E. Langmann and A.J. Niemi, Phys. Lett. B463 (1999) 252; Y.M. Cho, H.W. Lee and D.G. Pak, Phys. Lett. B525 (2002) 347

[13] S.E. Barnes, J. Phys. F6 (1976) 1375; L.D. Faddeev and L.A. Takhtajan, Phys. Lett. A85 (1981) 375; P. Coleman, Phys. Rev. B29 (1984) 3035

[14] G. Baskaran and P.W. Andersson, Phys. Rev. B37 (1988) 580; P.W. Andersson, Science 235 (1987) 1196; for a review see P.A. Lee, N. Nagaosa and X.-G. Wen, cond-mat/0410445

[15] A.J. Niemi and N. Walet, Phys. Rev. D72 (2005) 054007

[16] M.N. Chernodub and A.J. Niemi, quant-ph/0604162

[17] K.S.S. Stelle, Phys. Rev. D16 (1977) 953; E.S. Fradkin and A.A. Tseytlin, Phys. Lett. 
104B (1981) 377; E.S. Fradkin and A.A. Tseytlin, Phys. Rept. 119 (1985) 233

[18] M.N. Chernodub and M.I. Polikarpov, in Confinement, duality, and nonperturbative aspects of QCD, P. van Baal, Ed. (Plenum Press, New York 1998) (hep-th/9710205); T. Suzuki, Prog. Theor. Phys. Suppl. 131, 633 (1998); R.W. Haymaker, Phys. Rept. 315, $153(1999)$.

[19] K.-I. Kondo, Phys. Lett. B514 (2001) 335

[20] A.J. Niemi, JHEP 0408 (2004) 035

[21] F.V. Gubarev, L. Stodolsky and V.I. Zakharov, Phys. Rev. Lett. 86, 2220 (2001); L Stodolsky, P. van Baal and V.I. Zakharov, Phys. Lett. B552, 214 (2003).

[22] E. Babaev, L.D. Faddeev and A.J. Niemi, Phys. Rev. B65, 100512(R) (2002)

[23] G. ’t Hooft, Nucl. Phys. B 79, 276 (1974)

[24] S.V. Shabanov, Phys. Lett. B463 (1999) 263

[25] G.W. Gibbons, S.W. Hawking and M.J. Perry, Nucl. Phys. B138 (1978) 141 